\title{
Local Well-Posedness and Blow-Up for the Solutions to the Axisymmetric Inviscid Hall-MHD Equations
}

\author{
Eunji Jeong, Junha Kim, and Jihoon Lee \\ Department of Mathematics, Chung-Ang University, Seoul 06974, Republic of Korea \\ Correspondence should be addressed to Jihoon Lee; jhleepde@cau.ac.kr
}

Received 16 July 2018; Accepted 26 September 2018; Published 1 November 2018

Academic Editor: Carlo Bianca

Copyright (C) 2018 Eunji Jeong et al. This is an open access article distributed under the Creative Commons Attribution License, which permits unrestricted use, distribution, and reproduction in any medium, provided the original work is properly cited.

In this paper, we consider the regularity problem of the solutions to the axisymmetric, inviscid, and incompressible Hallmagnetohydrodynamics (Hall-MHD) equations. First, we obtain the local-in-time existence of sufficiently regular solutions to the axisymmetric inviscid Hall-MHD equations without resistivity. Second, we consider the inviscid axisymmetric Hall equations without fluids and prove that there exists a finite time blow-up of a classical solution due to the Hall term. Finally, we obtain some blow-up criteria for the axisymmetric resistive and inviscid Hall-MHD equations.

\section{Introduction}

Magnetohydrodynamics is the study of the dynamics of the electrically conducting fluids. The dynamics of the fluids can be described by the Navier-Stokes equations and the dynamics of the magnetic field can be described by the Maxwell equations for a perfect conductor. The Hall-magnetohydrodynamics (Hall-MHD) equations differ from the standard incompressible MHD equations by the Hall term $\nabla \times((\nabla \times B) \times B)$, which plays an important role in the study of the magnetic reconnection in the case of the large magnetic shear (see $[1,2])$. In [3], Hall-MHD equations have been formally derived from using the generalized Ohm's law instead of the usual simplified Ohm's law. The Cauchy problem for threedimensional incompressible Hall-MHD equations reads as follows:

$$
\begin{aligned}
& \partial_{t} u+(u \cdot \nabla) u+\nabla p=(\nabla \times B) \times B+v \Delta u, \\
& \nabla \cdot u=0, \quad \text { in } \mathbb{R}^{3} \times(0, \infty), \\
& \partial_{t} B-\nabla \times(u \times B)+\nabla \times((\nabla \times B) \times B)=\kappa \Delta B, \\
& (u(0, x), B(0, x))=\left(u_{0}(x), B_{0}(x)\right), \quad \text { in } \mathbb{R}^{3},
\end{aligned}
$$

where $u, B$, and $p$ represent three-dimensional velocity vector field, the magnetic field, and scalar pressure, respectively. The initial data $u_{0}$ and $B_{0}$ satisfy

$$
\nabla \cdot u_{0}=\nabla \cdot B_{0}=0 .
$$

Note that if $\nabla \cdot B_{0}=0$, then the divergence free condition is propagated by $(1)_{3}$. We only consider $\mathbb{R}^{3}$ for a spatial domain with vanishing at infinity condition for simplicity.

The Hall magnetohydrodynamics were studied systematically by Lighthill [2]. The Hall-MHD is important, describing many physical phenomena, e.g., space plasmas, star formation, neutron stars, and geo-dynamo (see $[1,4-8]$ and references therein).

The Hall-MHD equations have been mathematically investigated in several works. In [9], Acheritogaray, Degond, Frouvelle, and Liu derived the Hall-MHD equations from either two fluids' model or kinetic models in a mathematically more rigorous way. In [10], the global existence of weak solutions to (1) and the local well-posedness of classical solution are established when $\nu, \kappa>0$. Also, a blow-up criterion for smooth solution to (1) and the global existence of smooth solution for small initial data are obtained (see [10, Theorem 2.2 and 2.3]). Some of the results have been refined by many authors (see [11-13] and references therein). Recently, temporal decay for the weak solution and smooth 
solution with small data to Hall-MHD are also established in [14]. Spatial and temporal decays of solutions to (1) have been investigated in [15].

Using vector identity, we can rewrite (1) as follows:

$$
\begin{aligned}
\partial_{t} u+(u \cdot \nabla) u+\nabla\left(p+\frac{|B|^{2}}{2}\right) & =(B \cdot \nabla) B+v \Delta u, \\
\nabla \cdot u= & 0, \\
& \text { in } \mathbb{R}^{3} \times(0, \infty), \\
\partial_{t} B+(u \cdot \nabla) B+\nabla \times((\nabla \times B) \times B)= & (B \cdot \nabla) u+\kappa \Delta B, \\
(u(0, x), B(0, x))= & \left(u_{0}(x), B_{0}(x)\right),
\end{aligned}
$$$$
\text { in } \mathbb{R}^{3} \text {. }
$$

Note that a weak solution $(u, B)$ to $(1)$ satisfies the following energy inequality (see [10]):

$$
\begin{aligned}
& \|u(t)\|_{L^{2}}^{2}+\|B(t)\|_{L^{2}}^{2} \\
& \quad+2 \int_{0}^{t} v\|\nabla u(\cdot, \tau)\|_{L^{2}}^{2}+\kappa\|\nabla B(\cdot, \tau)\|_{L^{2}}^{2} d \tau \\
& \leq\left\|u_{0}\right\|_{L^{2}}^{2}+\left\|B_{0}\right\|_{L^{2}}^{2},
\end{aligned}
$$

for almost every $t \in[0, \infty)$.

Next we consider the mathematical setting for the axisymmetric Hall-MHD equations. Introducing the cylindrical coordinates

$$
\begin{aligned}
& r=\sqrt{x_{1}^{2}+x_{2}^{2}}, \\
& \theta=\arctan \frac{x_{2}}{x_{1}}, \\
& z=x_{3},
\end{aligned}
$$

and standard basis vectors for the cylindrical coordinates

$$
\begin{aligned}
& e_{r}=(\cos \theta, \sin \theta, 0), \\
& e_{\theta}=(-\sin \theta, \cos \theta, 0), \\
& e_{z}=(0,0,1),
\end{aligned}
$$

we set

$$
\begin{aligned}
& u=u^{r}(r, z, t) e_{r}+u^{z}(r, z, t) e_{z}, \\
& B=B^{\theta}(r, z, t) e_{\theta} .
\end{aligned}
$$

It is well-known that the local-in-time classical solutions to axisymmetric Navier-Stokes equations without swirl persist to any time (see $[16,17])$. But the global well-posedness for the axisymmetric Navier-Stokes equations with swirl component is widely open and has been one of the most fundamental open problems in the Navier-Stokes equations.
The axisymmetric MHD equations can be written as follows:

$$
\begin{aligned}
& \partial_{t} u^{r}+\left(u^{r} \partial_{r}+u^{z} \partial_{z}\right) u^{r}-v\left(\partial_{r}^{2}+\frac{1}{r} \partial_{r}+\partial_{z}^{2}-\frac{1}{r^{2}}\right) u^{r} \\
& +\partial_{r} P=-\frac{\left(B^{\theta}\right)^{2}}{r}, \\
& \partial_{t} u^{z}+\left(u^{r} \partial_{r}+u^{z} \partial_{z}\right) u^{z}-v\left(\partial_{r}^{2}+\frac{1}{r} \partial_{r}+\partial_{z}^{2}\right) u^{z} \\
& \quad+\partial_{z} P=0, \\
& \partial_{r} u^{r}+\frac{u^{r}}{r}+\partial_{z} u^{z}=0, \\
& \partial_{t} B^{\theta}+\left(u^{r} \partial_{r}+u^{z} \partial_{z}\right) B^{\theta}-\frac{u^{r} B^{\theta}}{r} \\
& \quad-\kappa\left(\partial_{r}^{2}+\frac{1}{r} \partial_{r}+\partial_{z}^{2}-\frac{1}{r^{2}}\right) B^{\theta}=0 .
\end{aligned}
$$

Lei [18] proved the global well-posedness of classical solutions to system (8) when $\kappa \geq 0$.

Then axisymmetric Hall-MHD equations are reduced to the following:

$$
\begin{aligned}
& \partial_{t} u^{r}+\left(u^{r} \partial_{r}+u^{z} \partial_{z}\right) u^{r}-v\left(\partial_{r}^{2}+\frac{1}{r} \partial_{r}+\partial_{z}^{2}-\frac{1}{r^{2}}\right) u^{r} \\
& +\partial_{r} P=-\frac{\left(B^{\theta}\right)^{2}}{r}, \\
& \partial_{t} u^{z}+\left(u^{r} \partial_{r}+u^{z} \partial_{z}\right) u^{z}-v\left(\partial_{r}^{2}+\frac{1}{r} \partial_{r}+\partial_{z}^{2}\right) u^{z} \\
& +\partial_{z} P=0, \\
& \partial_{r} u^{r}+\frac{u^{r}}{r}+\partial_{z} u^{z}=0, \\
& \partial_{t} B^{\theta}+\left(u^{r} \partial_{r}+u^{z} \partial_{z}\right) B^{\theta}-\frac{u^{r} B^{\theta}}{r} \\
& \quad-\kappa\left(\partial_{r}^{2}+\frac{1}{r} \partial_{r}+\partial_{z}^{2}-\frac{1}{r^{2}}\right) B^{\theta}=\partial_{z}\left(\frac{\left(B^{\theta}\right)^{2}}{r}\right) .
\end{aligned}
$$

For axisymmetric Hall-MHD equations, the global wellposedness of the axisymmetric solutions to the viscous case $(\nu, \kappa>0)$ was first established by Fan, Huang, and Nakamura [19]. Recently, Chae and Weng [20] showed that the incompressible Hall-MHD system without resistivity is not globally in time well-posed in any Sobolev space $H^{m}\left(\mathbb{R}^{3}\right)$ with $m>7 / 2$. But local-in-time existence of smooth solution to (1) is totally open when $\kappa \equiv 0$. Compared with the work in [18], it seems very surprising that Hall term plays a dominant role for the occurrence of the singularity and even for the local well-posedness of the partially viscous Hall-MHD problems. In this paper, we intend to investigate the blow-up problem for the solutions to the partially viscous axisymmetric HallMHD equations and local-in-time existence of solutions to 
such solution with the axisymmetry. Setting $\omega^{\theta}=\partial_{z} u^{r}-\partial_{r} u^{z}$, $\Omega=\omega^{\theta} / r$, and $\Pi=B^{\theta} / r$, (9) are equivalent to the following equations:

$$
\begin{gathered}
\frac{\partial \Omega}{\partial t}+\left(u^{r} \partial_{r}+u^{z} \partial_{z}\right) \Omega+2 \Pi \partial_{z} \Pi \\
=v\left(\partial_{r}^{2}+\frac{3}{r} \partial_{r}+\partial_{z}^{2}\right) \Omega, \\
\frac{\partial \Pi}{\partial t}+\left(u^{r} \partial_{r}+u^{z} \partial_{z}\right) \Pi-2 \Pi \partial_{z} \Pi \\
=\kappa\left(\partial_{r}^{2}+\frac{3}{r} \partial_{r}+\partial_{z}^{2}\right) \Pi .
\end{gathered}
$$

$\begin{aligned} \frac{\operatorname{curl} u}{r} & =\Omega, \\ \frac{B}{r} & =\Pi,\end{aligned}$

$\Omega(t) \in C\left(\left[0, T_{0}\right) ; H^{m}\right) \cap \operatorname{Lip}\left(\left[0, T_{0}\right) ; H^{m-1}\right)$ and $u(t), B(t), \Pi(t) \in C\left(\left[0, T_{0}\right) ; H^{m}\right) \cap \operatorname{Lip}\left(\left[0, T_{0}\right) ; H^{m-1}\right)$.

Remark 2. Since the local-in-time regularity of solution to (1) is necessary to preserve the axisymmetry of the HallMHD equations locally in time, Theorem 1 cannot resolve the open question raised from [20]. We remark that the relation between (11)-(12) and (1) cannot be justified without local well-posedness of solution to $(1)(\kappa=\nu \equiv 0)$.

Next, we consider the local well-posedness/blow-up problem for the axisymmetric Hall equations with zero fluid velocity and $\kappa=0$. We rewrite the Hall equation for $\Pi=B^{\theta} / r$ :

$$
\begin{aligned}
\partial_{t} \Pi & =-2 \Pi \partial_{z} \Pi, \\
\Pi(x, 0) & =\Pi_{0}(x) .
\end{aligned}
$$

The above equation has similar features to the inviscid Burgers equation.

Theorem 3. Assume $\Pi_{0} \in H^{m}\left(\mathbb{R}^{3}\right)$ for any integer $m>5 / 2$. Then there exist $T_{0}>0$ and a classical solution to (14) such that

$$
\begin{aligned}
\Pi(t) \in & C\left(\left[0, T_{0}\right) ; H^{m}\left(\mathbb{R}^{3}\right)\right) \\
& \cap \operatorname{Lip}\left(\left[0, T_{0}\right) ; H^{m-1}\left(\mathbb{R}^{3}\right)\right) .
\end{aligned}
$$

Furthermore, for any $\Pi_{0} \neq 0$, there exists $T^{*}>0$ such that the above local solution $\Pi(t)$ has singularity at a finite time $t=T^{*}$.

Remark 4. In [20], the authors showed that if the initial data $\Pi_{0}$ satisfies $\partial_{z} \Pi_{0}(0,0) \geq 10^{4} C_{*}^{2}$ for some constant $C_{*}$ and $\Pi_{0}(0,0)>0$, then the singularity of $\Pi$ and $\Omega$ to axisymmetric inviscid Hall-MHD equations happens in a finite time. Theorem 3 implies that the singularity of $\Pi$ which is a solution to (14) happens in a finite time without any restriction of the initial data.
First, we consider the local well-posedness of the axisymmetric Hall MHD equations with $\nu \equiv 0$ and $\kappa \equiv 0$, and (10) can be rewritten as the equations

$$
\begin{gathered}
\partial_{t} \Pi+\left(u^{r} \partial_{r}+u^{z} \partial_{z}\right) \Pi=2 \Pi \partial_{z} \Pi \\
\partial_{t} \Omega+\left(u^{r} \partial_{r}+u^{z} \partial_{z}\right) \Omega=-2 \Pi \partial_{z} \Pi .
\end{gathered}
$$

Theorem 1. Let $\left(u_{0}, B_{0}, B_{0} / r\right.$, curl $\left.u_{0} / r\right) \in H^{m}\left(\mathbb{R}^{3}\right) \times$ $H^{m}\left(\mathbb{R}^{3}\right) \times H^{m}\left(\mathbb{R}^{3}\right) \times H^{m-1}\left(\mathbb{R}^{3}\right)$ with integer $m>9 / 2$ be axisymmetric initial data. There exist $T_{0}>0$ and classical and axisymmetric solution $(\Pi, \Omega)$ to (11)-(12) such that
Finally, we consider the incompressible Hall-MHD equations with zero fluid viscosity, for simplicity, assuming that $\nu \equiv 0$ and $\kappa \equiv 1$.

For the solutions to (10), global a priori bounds can be obtained; that is,

$$
\|\Pi\|_{L^{\infty}\left(0, T ; L^{\infty}\right)}+\|\Omega\|_{L^{\infty}\left(0, T ; L^{2}\right)}<\infty \quad \text { for all } T>0 .
$$

We assume that our initial data $\left(u_{0}, B_{0}\right)$ is axisymmetric and satisfies

$$
\begin{gathered}
\left(u_{0}, B_{0}, \Omega_{0}, \Pi_{0}\right) \in H^{m}\left(\mathbb{R}^{3}\right)^{4} \quad \text { with } m>\frac{5}{2}, \\
\nabla \cdot u_{0}=\nabla \cdot B_{0}=0 .
\end{gathered}
$$

The local-in-time existence of a smooth solution to (1) was already obtained by Chae, Wan, and Wu [21]. We obtain the following blow-up criterion for the local-in-time solutions to the Hall-MHD equations with $\nu \equiv 0$ and $\kappa \equiv 1$.

Theorem 5. Let $(u, B, p)$ be a local-in-time classical solution to the axisymmetric Hall-MHD equations (9) with $\nu=0, \kappa=1$. Then, for the first blow-up time $T^{*}<\infty$ of the classical solution to (9), it holds that

$$
\begin{aligned}
& \underset{t>T^{*}}{\limsup }\left(\|u(t)\|_{H^{m}}^{2}+\|B(t)\|_{H^{m}}^{2}+\|\Omega(t)\|_{H^{m}}^{2}\right. \\
& \left.\quad+\|\Pi(t)\|_{H^{m}}^{2}\right)=\infty,
\end{aligned}
$$

if and only if one of the following conditions holds:

(i)

$$
\int_{0}^{T^{*}}\left\|\frac{u_{+}^{r}}{r}\right\|_{L^{\infty}\left(\mathbb{R}^{3} \backslash C_{R}\right)} d t+\int_{0}^{T^{*}}\left\|r u_{+}^{r}\right\|_{L^{\infty}\left(C_{R}\right)} d t=\infty .
$$


(ii)

$$
\int_{0}^{T^{*}}\left\|\omega^{\theta}\right\|_{L^{2}\left(\mathbb{R}^{3} \mid C_{R}\right)}^{4} d t=\infty
$$

In the above, $C_{R}$ denotes the inside of infinite cylinder such that $C_{R}=\left\{(x, y, z) \mid x^{2}+y^{2}<R^{2}\right\}$ for any $R>0$ and $f_{+}(x)$ is defined by $\max \{f(x), 0\}$.

Remark 6. For the usual MHD equations, Lei [18] proved the global well-posedness for the axisymmetric MHD equations even for the case that $\nu \equiv 1$ and $\kappa \equiv 0$. For HallMHD equations, even local well-posedness is widely open for this zero resisitivity case due to the Hall term (see [20]). Theorem 5 indicates that if there exists a finite time singularity to the axisymmetric equations with $\nu \equiv 0$ and $\kappa \equiv 1$, then some norms of velocity and vorticity should approach infinity even for the outside of any infinite cylinder.

For simplicity, we denote $C$ for the harmless constant which changes from line to line, and $\|\cdot\|_{m}$ for $H^{m}$-norm.

\section{Proof of Theorem 1: Local-in-Time Existence}

In this section, we consider the local-in-time existence of regular solution to (11)-(12). Even if this problem does not seem complicated, we have a few technical difficulties raised from the axisymmetry; e.g., mollifying equations do not preserve the axial symmetry. We briefly explain some steps to prove Theorem 1: First, we consider system (21) without giving any symmetry. We can obtain the regularized system (25) by using standard mollifier. Then we can obtain various estimates and local-in-time existence of a solution for (21). Finally, we consider the initial data which is axial symmetry and axisymmetry is also preserved by (21) and this argument gives a proof of local-in-time existence of solution to (11)-(12).

We consider the equations

$$
\begin{aligned}
& \partial_{t} \omega+(\mathbf{u} \cdot \nabla) \omega=u^{r} \Omega-2 B \partial_{z} \Pi, \\
& \partial_{t} B+(\mathbf{u} \cdot \nabla) B=u^{r} \Pi+2 B \partial_{z} \Pi, \\
& \partial_{t} \Pi+(\mathbf{u} \cdot \nabla) \Pi=2 \Pi \partial_{z} \Pi, \\
& \partial_{t} \Omega+(\mathbf{u} \cdot \nabla) \Omega=-2 \Pi \partial_{z} \Pi,
\end{aligned}
$$

where $\omega, B, \Pi$, and $\Omega$ are assumed to be independent scalar valued functions without assuming symmetry for a while, and the divergence free velocity field $\mathbf{u}=u^{r}(r, \theta, z) e_{r}+$ $u^{z}(r, \theta, z) e_{z}$ is assumed to be obtained from the equation

$$
-\Delta \mathbf{u}=\nabla \times\left(\omega e_{\theta}\right) .
$$

Thus, we have

$$
\mathbf{u}(x)=\Phi *\left(\nabla \times\left(\omega e_{\theta}\right)\right), \quad \text { where } \Phi(x)=\frac{1}{4 \pi|x|} .
$$

If $\omega \in H^{1}\left(\mathbb{R}^{3}\right) \cap C\left(\mathbb{R}^{3}\right)$, then the divergence theorem and trace theorem induce the following estimates:

$$
\begin{aligned}
|\mathbf{u}(x)| \leq & \|\Phi\|_{L^{2}(B(0 ; 1))}\|\nabla \omega\|_{L^{2}\left(\mathbb{R}^{3}\right)} \\
& +\|\nabla \Phi\|_{L^{2}\left(\mathbb{R}^{3} \backslash B(0 ; 1)\right)}\|\omega\|_{L^{2}\left(\mathbb{R}^{3}\right)} \\
& +\int_{\partial B(0 ; 1)} \Phi(y) \omega(x-y) d S \\
\leq & \|\Phi\|_{L^{2}(B(0 ; 1))}\|\nabla \omega\|_{L^{2}\left(\mathbb{R}^{3}\right)} \\
& +\|\nabla \Phi\|_{L^{2}\left(\mathbb{R}^{3} \backslash B(0 ; 1)\right)}\|\omega\|_{L^{2}\left(\mathbb{R}^{3}\right)} \\
& +C\left(\int_{\partial B(0 ; 1)}|\omega(x-y)|^{2} d S\right)^{1 / 2} \\
\leq & \|\Phi\|_{L^{2}(B(0 ; 1))}\|\nabla \omega\|_{L^{2}\left(\mathbb{R}^{3}\right)} \\
& +\|\nabla \Phi\|_{L^{2}\left(\mathbb{R}^{3} \backslash B(0 ; 1)\right)}\|\omega\|_{L^{2}\left(\mathbb{R}^{3}\right)}+C\|\omega\|_{H^{1}\left(\mathbb{R}^{3}\right)} \\
\leq & C\|\omega\|_{H^{1}\left(\mathbb{R}^{3}\right)} .
\end{aligned}
$$

We define a regularized system of (21) as follows:

$$
\begin{aligned}
& \partial_{t} \omega_{\epsilon}+\mathscr{J}_{\epsilon}\left(\mathscr{F}_{\epsilon} \mathbf{u} \cdot \nabla\right) \mathscr{F}_{\epsilon} \omega_{\epsilon} \\
& \quad=\mathscr{F}_{\epsilon}\left(\mathscr{F}_{\epsilon} u_{\epsilon}^{r} \mathscr{F}_{\epsilon} \Omega_{\epsilon}\right)-2 \mathscr{F}_{\epsilon}\left(\mathscr{F}_{\epsilon} B_{\epsilon} \partial_{z} \mathscr{F}_{\epsilon} \Pi_{\epsilon}\right), \\
& \partial_{t} B_{\epsilon}+\mathscr{J}_{\epsilon}\left(\mathscr{F}_{\epsilon} \mathbf{u} \cdot \nabla\right) \mathscr{J}_{\epsilon} B_{\epsilon} \\
& \quad=\mathscr{F}_{\epsilon}\left(\mathscr{F}_{\epsilon} u_{\epsilon}^{r} \mathscr{F}_{\epsilon} \Pi_{\epsilon}\right)+2 \mathscr{F}_{\epsilon}\left(\mathscr{F}_{\epsilon} B_{\epsilon} \partial_{z} \mathscr{F}_{\epsilon} \Pi_{\epsilon}\right), \\
& \partial_{t} \Pi_{\epsilon}+\mathscr{F}_{\epsilon}\left(\mathscr{F}_{\epsilon} \mathbf{u} \cdot \nabla\right) \mathscr{F}_{\epsilon} \Pi_{\epsilon}=2 \mathscr{F}_{\epsilon}\left(\mathscr{F}_{\epsilon} \Pi_{\epsilon} \partial_{z} \mathscr{F}_{\epsilon} \Pi_{\epsilon}\right), \\
& \partial_{t} \Omega_{\epsilon}+\mathscr{F}_{\epsilon}\left(\mathscr{F}_{\epsilon} \mathbf{u} \cdot \nabla\right) \mathscr{F}_{\epsilon} \Omega_{\epsilon}=-2 \mathscr{F}_{\epsilon}\left(\mathscr{F}_{\epsilon} \Pi_{\epsilon} \partial_{z} \mathscr{F}_{\epsilon} \Pi_{\epsilon}\right),
\end{aligned}
$$

where $\mathscr{F}_{\epsilon}$ is a standard mollifier as in [22]. Next, we obtain apriori estimates to derive a time $T_{0}$ which does not depend on $\epsilon>0$. Then we prove that (25) have a local-intime solution $S_{\epsilon}=\left(\omega_{\epsilon}, B_{\epsilon}, \Pi_{\epsilon}, \Omega_{\epsilon}\right) \in C^{1}\left(0, T_{0} ; H^{m}\right) \times$ $C^{1}\left(0, T_{0} ; H^{m}\right) \times C^{1}\left(0, T_{0} ; H^{m+1}\right) \times C^{1}\left(0, T_{0} ; H^{m}\right)$ space for each $\epsilon>0$.

\section{Proposition 7. Let}

$$
\left\|S_{\epsilon}\right\|_{X^{m}}(t):=\left\|\omega_{\epsilon}\right\|_{m}+\left\|B_{\epsilon}\right\|_{m}+\left\|\Pi_{\epsilon}\right\|_{m+1}+\left\|\Omega_{\epsilon}\right\|_{m}
$$

where $X^{m}=H^{m}\left(\mathbb{R}^{3}\right) \times H^{m}\left(\mathbb{R}^{3}\right) \times H^{m+1}\left(\mathbb{R}^{3}\right) \times H^{m}\left(\mathbb{R}^{3}\right)$ with an integer $m>5 / 2$. Then, for some positive constant $C_{0}$ and $T<1 / C_{0} S_{0}$ with $S_{0}=\left\|S_{\epsilon}(0)\right\|_{X^{m}}$, we have

$$
\begin{gathered}
\sup _{0<t<T}\left\|S_{\epsilon}\right\|_{X^{m}}(t) \leq \frac{S_{0}}{1-C_{0} S_{0} T}, \\
\sup _{0<t<T}\left\|\partial_{t} S_{\epsilon}\right\|_{X^{m-1}}(t) \leq\left(\frac{S_{0}}{1-C_{0} S_{0} T}\right)^{2} .
\end{gathered}
$$


Proof. For $m>5 / 2$ and $1 \leq s \leq m$, we have the following inequality by the calculus inequality and Hölder's inequality:

$$
\begin{aligned}
& \frac{1}{2} \frac{d}{d t}\left\|\nabla^{s} \omega_{\epsilon}\right\|_{L^{2}}^{2} \\
& \quad \leq \int_{\mathbb{R}^{3}}\left|\mathscr{J}_{\epsilon} \nabla^{s} \omega_{\epsilon}\right|\left|\nabla^{s-1}\left(\mathscr{J}_{\epsilon} \nabla \mathbf{u}_{\epsilon} \cdot \mathscr{J}_{\epsilon} \nabla \omega_{\epsilon}\right)\right| d x \\
& \quad+\int_{\mathbb{R}^{3}}\left|\mathscr{J}_{\epsilon} \nabla^{s} \omega_{\epsilon}\right|\left|\nabla^{s}\left(\mathscr{J}_{\epsilon} u_{\epsilon}^{r} \mathscr{F}_{\epsilon} \Omega_{\epsilon}\right)\right| d x \\
& \quad+\int_{\mathbb{R}^{3}}\left|\mathscr{J}_{\epsilon} \nabla^{s} \omega_{\epsilon}\right|\left|\nabla^{s}\left(\mathscr{J}_{\epsilon} B_{\epsilon} \partial_{z} \mathscr{J}_{\epsilon} \Pi_{\epsilon}\right)\right| d x \leq\left\|\omega_{\epsilon}\right\|_{m} \\
& \left.\quad+\left\|\nabla \mathbf{u}_{\epsilon}\right\|_{L^{\infty}}\left\|\nabla^{s} \omega_{\epsilon}\right\|_{L^{2}}+\left\|\nabla^{s} \mathbf{u}_{\epsilon}\right\|_{L^{2}}\left\|\nabla \omega_{\epsilon}\right\|_{L^{\infty}}\right) \\
& \quad+\left\|\omega_{\epsilon}\right\|_{m}\left(\left\|u_{\epsilon}^{r}\right\|_{L^{\infty}}\left\|\nabla^{s} \Omega_{\epsilon}\right\|_{L^{2}}+\left\|\Omega_{\epsilon}\right\|_{L^{\infty}}\left\|\nabla^{s} u_{\epsilon}^{r}\right\|_{L^{2}}\right) \\
& \quad+2\left\|\omega_{\epsilon}\right\|_{m}\left(\left\|B_{\epsilon}\right\|_{L^{\infty}}\left\|\nabla^{s+1} \Pi_{\epsilon}\right\|_{L^{2}}\right. \\
& \left.\quad+\left\|\nabla \Pi_{\epsilon}\right\|_{L^{\infty}}\left\|\nabla^{s} B_{\epsilon}\right\|_{L^{2}}\right) \leq C\left\|\omega_{\epsilon}\right\|_{m}\left(\left\|\omega_{\epsilon}\right\|_{m-1}\left\|\omega_{\epsilon}\right\|_{m}\right. \\
& \left.\quad+\left\|\Omega_{\epsilon}\right\|_{m}\left\|\omega_{\epsilon}\right\|_{m}+\left\|\Pi_{\epsilon}\right\|_{m+1}\left\|B_{\epsilon}\right\|_{m}\right) .
\end{aligned}
$$

Similarly, for $m>5 / 2$ and $1 \leq s \leq m$, we can obtain

$$
\begin{gathered}
\frac{1}{2} \frac{d}{d t}\left\|\nabla^{s} B_{\epsilon}\right\|_{L^{2}}^{2} \leq\left\|B_{\epsilon}\right\|_{m}\left(\left\|\omega_{\epsilon}\right\|_{m-1}\left\|B_{\epsilon}\right\|_{m}\right. \\
\left.+\left\|\omega_{\epsilon}\right\|_{m}\left\|\Pi_{\epsilon}\right\|_{m}+\left\|\Pi_{\epsilon}\right\|_{m+1}\left\|B_{\epsilon}\right\|_{m}\right),
\end{gathered}
$$

and $1 \leq s \leq m+1$,

$$
\begin{aligned}
& \frac{1}{2} \frac{d}{d t}\left\|\nabla^{s} \Pi_{\epsilon}\right\|_{L^{2}}^{2} \\
& \quad \leq\left\|\Pi_{\epsilon}\right\|_{m+1}\left(\left\|\omega_{\epsilon}\right\|_{m}\left\|\Pi_{\epsilon}\right\|_{m+1}+\left\|\Pi_{\epsilon}\right\|_{m+1}^{2}\right) .
\end{aligned}
$$

For an integer $m>5 / 2$ and all integers $1 \leq s \leq m$, we have

$$
\frac{1}{2} \frac{d}{d t}\left\|\nabla^{s}\left(\Pi_{\epsilon}+\Omega_{\epsilon}\right)\right\|_{L^{2}}^{2} \leq\left\|\Pi_{\epsilon}+\Omega_{\epsilon}\right\|_{m}^{2}\left\|\omega_{\epsilon}\right\|_{m-1} .
$$

Hence, we conclude that, for $m>5 / 2$,

$$
\begin{aligned}
& \frac{d}{d t}\left\|S_{\epsilon}\right\|_{X^{m}}(t) \\
& \quad \leq \frac{1}{2} C_{0}\left(\left\|\omega_{\epsilon}\right\|_{m}^{2}+\left\|B_{\epsilon}\right\|_{m}^{2}+\left\|\Pi_{\epsilon}\right\|_{m+1}^{2}+\left\|\Pi_{\epsilon}+\Omega_{\epsilon}\right\|_{m}^{2}\right) \\
& \quad \leq C_{0}\left\|S_{\epsilon}\right\|_{X^{m}}^{2}(t) .
\end{aligned}
$$

Inequality (32) implies that (27) holds true.

Proposition 8. Assume $C_{0}$ and $S_{0}$ are the same as in Proposition 7. Let $T_{0}=3 / 4 C_{0} S_{0}$. Then for given initial data $\omega_{0}, B_{0}, \Omega_{0} \in H^{m}\left(\mathbb{R}^{3}\right)$, and $\Pi_{0} \in H^{m+1}\left(\mathbb{R}^{3}\right)$ with an integer $m>5 / 2$, there exists a unique solution $\left(\omega_{\epsilon}, B_{\epsilon}, \Pi_{\epsilon}, \Omega_{\epsilon}\right)$ to regularized system (25) such that $\omega_{\epsilon}, B_{\epsilon}, \Omega_{\epsilon} \in C^{1}\left(0, T_{0} ; H^{m}\right)$, $\Pi_{\epsilon} \in C^{1}\left(0, T_{0} ; H^{m+1}\right)$.
Proof. We set

$$
\begin{aligned}
F_{\epsilon}^{1}\left(\omega_{\epsilon}, B_{\epsilon}, \Pi_{\epsilon}, \Omega_{\epsilon}\right)= & -\mathscr{F}_{\epsilon}\left(\mathscr{F}_{\epsilon} \mathbf{u}_{\epsilon} \cdot \nabla\right) \mathscr{F}_{\epsilon} \omega_{\epsilon} \\
& +\mathscr{J}_{\epsilon}\left(\mathscr{F}_{\epsilon} u_{\epsilon}^{r} \mathscr{F}_{\epsilon} \Omega_{\epsilon}\right) \\
& -2 \mathscr{F}_{\epsilon}\left(\mathscr{F}_{\epsilon} B_{\epsilon} \partial_{z} \mathscr{F}_{\epsilon} \Pi_{\epsilon}\right), \\
F_{\epsilon}^{2}\left(\omega_{\epsilon}, B_{\epsilon}, \Pi_{\epsilon}, \Omega_{\epsilon}\right)= & -\mathscr{F}_{\epsilon}\left(\mathscr{F}_{\epsilon} \mathbf{u}_{\epsilon} \cdot \nabla\right) \mathscr{F}_{\epsilon} B_{\epsilon} \\
& +\mathscr{F}_{\epsilon}\left(\mathscr{F}_{\epsilon} u_{\epsilon}^{r} \mathscr{F}_{\epsilon} \Pi_{\epsilon}\right) \\
& +2 \mathscr{F}_{\epsilon}\left(\mathscr{F}_{\epsilon} B_{\epsilon} \partial_{z} \mathscr{F}_{\epsilon} \Pi_{\epsilon}\right), \\
F_{\epsilon}^{3}\left(\omega_{\epsilon}, \Pi_{\epsilon}\right)= & -\mathscr{F}_{\epsilon}\left(\mathscr{F}_{\epsilon} \mathbf{u}_{\epsilon} \cdot \nabla\right) \mathscr{F}_{\epsilon} \Pi_{\epsilon} \\
& +2 \mathscr{F}_{\epsilon}\left(\mathscr{F}_{\epsilon} \Pi_{\epsilon} \partial_{z} \mathscr{F}_{\epsilon} \Pi_{\epsilon}\right), \\
F_{\epsilon}^{4}\left(\omega_{\epsilon}, \Pi_{\epsilon}, \Omega_{\epsilon}\right)= & -\mathscr{F}_{\epsilon}\left(\mathscr{F}_{\epsilon} \mathbf{u}_{\epsilon} \cdot \nabla\right) \mathscr{F}_{\epsilon} \Omega_{\epsilon} \\
& -2 \mathscr{F}_{\epsilon}\left(\mathscr{F}_{\epsilon} \Pi_{\epsilon} \partial_{z} \mathscr{F}_{\epsilon} \Pi_{\epsilon}\right) .
\end{aligned}
$$

First, we show that $F_{\epsilon}^{1}$ is Lipschitz continuous on $H^{m}$ space. We estimate for $\epsilon<1, m \geq 3$

$$
\begin{aligned}
& \left\|F_{\epsilon}^{1}\left(\omega_{\epsilon}, B_{\epsilon}, \Pi_{\epsilon}, \Omega_{\epsilon}\right)-F_{\epsilon}^{1}\left(\widetilde{\omega}_{\epsilon}, B_{\epsilon}, \Pi_{\epsilon}, \Omega_{\epsilon}\right)\right\|_{m} \\
& \quad \leq\left\|\left(\mathscr{F}_{\epsilon}\left(\mathbf{u}_{\epsilon}-\widetilde{\mathbf{u}}_{\epsilon}\right) \cdot \nabla\right) \mathscr{F}_{\epsilon} \omega_{\epsilon}\right\|_{m} \\
& +\left\|\left(\mathscr{F}_{\epsilon} \widetilde{\mathbf{u}}_{\epsilon} \cdot \nabla\right)\left(\mathscr{J}_{\epsilon} \omega_{\epsilon}-\mathscr{J}_{\epsilon} \widetilde{\omega}_{\epsilon}\right)\right\|_{m} \\
& \quad+\left\|\mathscr{F}_{\epsilon}\left(u_{\epsilon}^{r}-\widetilde{u}_{\epsilon}^{r}\right) \mathscr{F}_{\epsilon} \Omega_{\epsilon}\right\|_{m} \\
& \leq \frac{C}{\epsilon}\left(\left\|\mathbf{u}_{\epsilon}-\widetilde{\mathbf{u}}_{\epsilon}\right\|_{L^{\infty}}+\left\|\omega_{\epsilon}-\widetilde{\omega}_{\epsilon}\right\|_{m-1}\right) \\
& \cdot\left(\left\|\omega_{\epsilon}\right\|_{m}+\epsilon\left\|\Omega_{\epsilon}\right\|_{m}\right)+\frac{C}{\epsilon}\left(\left\|\widetilde{\mathbf{u}}_{\epsilon}\right\|_{L^{\infty}}+\left\|\widetilde{\omega}_{\epsilon}\right\|_{m-1}\right) \\
& \cdot\left\|\omega_{\epsilon}-\widetilde{\omega}_{\epsilon}\right\|_{m} \leq \frac{C}{\epsilon}\left(\left\|\omega_{\epsilon}\right\|_{m}+\left\|\Omega_{\epsilon}\right\|_{m}+\left\|\widetilde{\omega}_{\epsilon}\right\|_{m-1}\right)
\end{aligned}
$$$$
\cdot\left\|\omega_{\epsilon}-\widetilde{\omega}_{\epsilon}\right\|_{m} .
$$

By the similar estimates as in (34), we obtain

$$
\begin{aligned}
& \left\|F_{\epsilon}^{1}\left(\omega_{\epsilon}, B_{\epsilon}, \Pi_{\epsilon}, \Omega_{\epsilon}\right)-F_{\epsilon}^{1}\left(\omega_{\epsilon}, \widetilde{B}_{\epsilon}, \Pi_{\epsilon}, \Omega_{\epsilon}\right)\right\|_{m} \\
& \quad \leq 2\left\|\left(\mathscr{F}_{\epsilon} B_{\epsilon}-\mathscr{F}_{\epsilon} \widetilde{B}_{\epsilon}\right) \partial_{z} \mathscr{F}_{\epsilon} \Pi_{\epsilon}\right\|_{m} \\
& \quad \leq C\left\|\Pi_{\epsilon}\right\|_{m+1}\left\|B_{\epsilon}-\widetilde{B}_{\epsilon}\right\|_{m}, \\
& \left\|F_{\epsilon}^{1}\left(\omega_{\epsilon}, B_{\epsilon}, \Pi_{\epsilon}, \Omega_{\epsilon}\right)-F_{\epsilon}^{1}\left(\omega_{\epsilon}, B_{\epsilon}, \widetilde{\Pi}_{\epsilon}, \Omega_{\epsilon}\right)\right\|_{m} \\
& \quad \leq 2\left\|\mathscr{F}_{\epsilon} B_{\epsilon}\left(\partial_{z} \mathscr{F}_{\epsilon} \Pi_{\epsilon}-\partial_{z} \mathscr{F}_{\epsilon} \widetilde{\Pi}_{\epsilon}\right)\right\|_{m} \\
& \quad \leq C\left\|B_{\epsilon}\right\|_{m}\left\|\Pi_{\epsilon}-\widetilde{\Pi}_{\epsilon}\right\|_{m+1}, \\
& \left\|F_{\epsilon}^{1}\left(\omega_{\epsilon}, B_{\epsilon}, \Pi_{\epsilon}, \Omega_{\epsilon}\right)-F_{\epsilon}^{1}\left(\omega_{\epsilon}, B_{\epsilon}, \Pi_{\epsilon}, \widetilde{\Omega}_{\epsilon}\right)\right\|_{m} \\
& \leq\left\|\mathscr{F}_{\epsilon} u_{\epsilon}^{r}\left(\mathscr{F}_{\epsilon} \Omega_{\epsilon}-\mathscr{J}_{\epsilon} \widetilde{\Omega}_{\epsilon}\right)\right\|_{m} \\
& \leq C\left\|\omega_{\epsilon}\right\|_{m-1}\left\|\Omega_{\epsilon}-\widetilde{\Omega}_{\epsilon}\right\|_{m} .
\end{aligned}
$$


By the virtue of properties of mollifier, Lipschitz continuity of the remaining functions $F_{\epsilon}^{j}, j=2,3,4$, can be obtained with constant $C / \epsilon$. Thus, we can deduce the following for $\|$. $\left\|_{X^{m}},\right\| \cdot \|_{X^{m}} \leq R$

$$
\left\|F_{\epsilon}(\cdot)-F_{\epsilon}(\cdot)\right\|_{X^{m}} \leq \frac{C}{\epsilon} R\|\cdot-\tau\|_{X^{m}}
$$

with $m \geq 3$ and $F_{\epsilon}=\left(F_{\epsilon}^{1}, F_{\epsilon}^{2}, F_{\epsilon}^{3}, F_{\epsilon}^{4}\right)$. Now we use the Picard theorem with domain $X^{m}$. By picking any initial data $S_{\epsilon}(0) \epsilon$ $X^{m}$ and choosing $T_{0}=3 / 4 C_{0} S_{0}$, we have, for $R=S_{0} /(1-$ $\left.C_{0} T_{0} S_{0}\right)=4 S_{0}$,

$$
\begin{gathered}
\frac{d}{d t}\left\|S_{\epsilon}\right\|_{X^{m}}(t)=F_{\epsilon}\left(S_{\epsilon}(t)\right), \\
\left\|F_{\epsilon}\left(S_{\epsilon}\right)-F_{\epsilon}\left(\widetilde{S}_{\epsilon}\right)\right\|_{X^{m}} \leq \frac{4 C S_{0}}{\epsilon}\left\|S_{\epsilon}-\widetilde{S}_{\epsilon}\right\|_{X^{m}}
\end{gathered}
$$

where $\left\|S_{\epsilon}\right\|_{X^{m}},\left\|\widetilde{S}_{\epsilon}\right\|_{X^{m}} \leq 4 S_{0}$. Therefore, the Picard theorem implies that, for each $0<\epsilon<1$, there exists a unique solution $S_{\epsilon}(t) \in C^{1}\left(0, T_{\epsilon} ; X^{m}\right)$ for a fixed time $T_{\epsilon}>0$. For simplicity, let $T_{\epsilon}$ be the maximal existence time of such solution. Suppose that, for some $0<\epsilon<1$, we have $T_{\epsilon}<T_{0}$. Then by Proposition 7 , for arbitrarily small $\delta>0$, we have

$$
\left\|S_{\epsilon}\right\|_{X^{m}}\left(T_{\epsilon}-\delta\right) \leq \frac{S_{0}}{1-C_{0} S_{0} T_{\epsilon}}<4 S_{0} .
$$

If we apply the standard continuation argument, then we can have local-in-time solution $S_{\epsilon}$ at least until $T_{0}$. This contradicts the assumption that $T_{\epsilon}<T_{0}$. Hence we prove that, for any $0<\epsilon<1$, there is a unique solution $S_{\epsilon}(t)$ with a uniform time $T_{0}$, such that $S_{\epsilon}(t) \in C^{1}\left(0, T_{0} ; X^{m}\right)$. This completes the proof.

Proposition 9. For an integer $m>7 / 2$, the solutions obtained in Proposition 8 form the Cauchy sequences in the following spaces:

$$
\begin{array}{r}
\left\{S_{\epsilon}\right\} \in C\left(0, T_{0} ; X^{1}\right), \\
\left\{\partial_{t} S_{\epsilon}\right\} \in C\left(0, T_{0} ; X^{0}\right) .
\end{array}
$$

Proof. Taking $\partial_{i}$ operator $(i=1,2,3)$ on both sides of $(25)_{1}$ and multiplying $\partial_{i} \omega_{\epsilon}$, we deduce that

$$
\begin{aligned}
& \frac{1}{2} \frac{\partial}{\partial t}\left|\partial_{i}\left(\omega_{\epsilon}-\omega_{\epsilon^{\prime}}\right)\right|^{2} \\
& \quad=\partial_{i}\left(F_{\epsilon}^{1}\left(\omega_{\epsilon}, B_{\epsilon}, \Pi_{\epsilon}, \Omega_{\epsilon}\right)-F_{\epsilon^{\prime}}^{1}\left(\omega_{\epsilon^{\prime}}, B_{\epsilon^{\prime}}, \Pi_{\epsilon^{\prime}}, \Omega_{\epsilon^{\prime}}\right)\right) \\
& \cdot \partial_{i}\left(\omega_{\epsilon}-\omega_{\epsilon^{\prime}}\right)=A_{1}+A_{2}+A_{3}+A_{4}+A_{5}+A_{6},
\end{aligned}
$$

where

$A_{1}$

$$
\begin{aligned}
= & \left(\left(\mathscr{F}_{\epsilon}-\mathscr{J}_{\epsilon^{\prime}}\right) \partial_{i}\left(\omega_{\epsilon}-\omega_{\epsilon^{\prime}}\right)\right)\left(\mathscr{F}_{\epsilon} \partial_{i} \mathbf{u}_{\epsilon} \cdot \nabla\right) \mathscr{F}_{\epsilon} \omega_{\epsilon} \\
& +\left(\mathscr{F}_{\epsilon^{\prime}} \partial_{i}\left(\omega_{\epsilon}-\omega_{\epsilon^{\prime}}\right)\right)\left(\left(\mathscr{F}_{\epsilon}-\mathscr{F}_{\epsilon^{\prime}}\right) \partial_{i} \mathbf{u}_{\epsilon} \cdot \nabla\right) \mathscr{F}_{\epsilon} \omega_{\epsilon} \\
& +\left(\mathscr{F}_{\epsilon^{\prime}} \partial_{i}\left(\omega_{\epsilon}-\omega_{\epsilon^{\prime}}\right)\right)\left(\mathscr{F}_{\epsilon^{\prime}} \partial_{i}\left(\mathbf{u}_{\epsilon}-\mathbf{u}_{\epsilon^{\prime}}\right) \cdot \nabla\right) \mathscr{F}_{\epsilon} \omega_{\epsilon} \\
& +\left(\mathscr{F}_{\epsilon^{\prime}} \partial_{i}\left(\omega_{\epsilon}-\omega_{\epsilon^{\prime}}\right)\right)\left(\mathscr{F}_{\epsilon^{\prime}} \partial_{i} \mathbf{u}_{\epsilon^{\prime}} \cdot \nabla\right)\left(\mathscr{F}_{\epsilon}-\mathscr{F}_{\epsilon^{\prime}}\right) \omega_{\epsilon} \\
& +\left(\mathscr{F}_{\epsilon^{\prime}} \partial_{i}\left(\omega_{\epsilon}-\omega_{\epsilon^{\prime}}\right)\right)\left(\mathscr{F}_{\epsilon^{\prime}} \partial_{i} \mathbf{u}_{\epsilon^{\prime}} \cdot \nabla\right) \mathscr{J}_{\epsilon^{\prime}}\left(\omega_{\epsilon}-\omega_{\epsilon^{\prime}}\right),
\end{aligned}
$$

$A_{2}$

$$
\begin{aligned}
= & \left(\left(\mathscr{F}_{\epsilon}-\mathscr{F}_{\epsilon^{\prime}}\right) \partial_{i}\left(\omega_{\epsilon}-\omega_{\epsilon^{\prime}}\right)\right)\left(\mathscr{F}_{\epsilon} \mathbf{u}_{\epsilon} \cdot \nabla\right) \mathscr{F}_{\epsilon} \partial_{i} \omega_{\epsilon} \\
& +\left(\mathscr{F}_{\epsilon^{\prime}} \partial_{i}\left(\omega_{\epsilon}-\omega_{\epsilon^{\prime}}\right)\right)\left(\left(\mathscr{F}_{\epsilon}-\mathscr{F}_{\epsilon^{\prime}}\right) \mathbf{u}_{\epsilon} \cdot \nabla\right) \mathscr{F}_{\epsilon} \partial_{i} \omega_{\epsilon} \\
& +\left(\mathscr{F}_{\epsilon^{\prime}} \partial_{i}\left(\omega_{\epsilon}-\omega_{\epsilon^{\prime}}\right)\right)\left(\mathscr{F}_{\epsilon^{\prime}}\left(\mathbf{u}_{\epsilon}-\mathbf{u}_{\epsilon^{\prime}}\right) \cdot \nabla\right) \mathscr{F}_{\epsilon} \partial_{i} \omega_{\epsilon} \\
& +\left(\mathscr{F}_{\epsilon^{\prime}} \partial_{i}\left(\omega_{\epsilon}-\omega_{\epsilon^{\prime}}\right)\right)\left(\mathscr{F}_{\epsilon^{\prime}} \mathbf{u}_{\epsilon^{\prime}} \cdot \nabla\right)\left(\mathscr{F}_{\epsilon}-\mathscr{F}_{\epsilon^{\prime}}\right) \partial_{i} \omega_{\epsilon} \\
& +\left(\mathscr{F}_{\epsilon^{\prime}} \partial_{i}\left(\omega_{\epsilon}-\omega_{\epsilon^{\prime}}\right)\right)\left(\mathscr{F}_{\epsilon^{\prime}} \mathbf{u}_{\epsilon^{\prime}} \cdot \nabla\right) \mathscr{F}_{\epsilon^{\prime}} \partial_{i}\left(\omega_{\epsilon}-\omega_{\epsilon^{\prime}}\right),
\end{aligned}
$$

$A_{3}$

$$
\begin{aligned}
= & \left(\left(\mathscr{F}_{\epsilon}-\mathscr{F}_{\epsilon^{\prime}}\right) \partial_{i}\left(\omega_{\epsilon}-\omega_{\epsilon^{\prime}}\right)\right) \mathscr{F}_{\epsilon} \partial_{i} u_{\epsilon}^{r} \mathscr{F}_{\epsilon} \Omega_{\epsilon} \\
& +\left(\mathscr{F}_{\epsilon^{\prime}} \partial_{i}\left(\omega_{\epsilon}-\omega_{\epsilon^{\prime}}\right)\right)\left(\mathscr{F}_{\epsilon}-\mathscr{F}_{\epsilon^{\prime}}\right) \partial_{i} u_{\epsilon}^{r} \mathscr{F}_{\epsilon} \Omega_{\epsilon} \\
& +\left(\mathscr{F}_{\epsilon^{\prime}} \partial_{i}\left(\omega_{\epsilon}-\omega_{\epsilon^{\prime}}\right)\right) \mathscr{F}_{\epsilon^{\prime}} \partial_{i}\left(u_{\epsilon}^{r}-u_{\epsilon^{\prime}}^{r}\right) \mathscr{F}_{\epsilon} \Omega_{\epsilon} \\
& +\left(\mathscr{F}_{\epsilon^{\prime}} \partial_{i}\left(\omega_{\epsilon}-\omega_{\epsilon^{\prime}}\right)\right) \mathscr{J}_{\epsilon^{\prime}} \partial_{i} u_{\epsilon^{\prime}}^{r}\left(\mathscr{F}_{\epsilon}-\mathscr{J}_{\epsilon^{\prime}}\right) \Omega_{\epsilon} \\
& +\left(\mathscr{F}_{\epsilon^{\prime}} \partial_{i}\left(\omega_{\epsilon}-\omega_{\epsilon^{\prime}}\right)\right) \mathscr{F}_{\epsilon^{\prime}} \partial_{i} u_{\epsilon^{\prime}}^{r} \mathscr{F}_{\epsilon^{\prime}}\left(\Omega_{\epsilon}-\Omega_{\epsilon^{\prime}}\right),
\end{aligned}
$$

$A_{4}$

$$
\begin{aligned}
= & \left(\left(\mathscr{F}_{\epsilon}-\mathscr{F}_{\epsilon^{\prime}}\right) \partial_{i}\left(\omega_{\epsilon}-\omega_{\epsilon^{\prime}}\right)\right) \mathscr{F}_{\epsilon} u_{\epsilon}^{r} \mathscr{F}_{\epsilon} \partial_{i} \Omega_{\epsilon} \\
& +\left(\mathscr{F}_{\epsilon^{\prime}} \partial_{i}\left(\omega_{\epsilon}-\omega_{\epsilon^{\prime}}\right)\right)\left(\mathscr{F}_{\epsilon}-\mathscr{F}_{\epsilon^{\prime}}\right) u_{\epsilon}^{r} \mathscr{F}_{\epsilon} \partial_{i} \Omega_{\epsilon} \\
& +\left(\mathscr{F}_{\epsilon^{\prime}} \partial_{i}\left(\omega_{\epsilon}-\omega_{\epsilon^{\prime}}\right)\right) \mathscr{F}_{\epsilon^{\prime}}\left(u_{\epsilon}^{r}-u_{\epsilon^{\prime}}^{r}\right) \mathscr{F}_{\epsilon} \partial_{i} \Omega_{\epsilon} \\
& +\left(\mathscr{F}_{\epsilon^{\prime}} \partial_{i}\left(\omega_{\epsilon}-\omega_{\epsilon^{\prime}}\right)\right) \mathscr{F}_{\epsilon^{\prime}} u_{\epsilon^{\prime}}^{r}\left(\mathscr{F}_{\epsilon}-\mathscr{F}_{\epsilon^{\prime}}\right) \partial_{i} \Omega_{\epsilon} \\
& +\left(\mathscr{F}_{\epsilon^{\prime}} \partial_{i}\left(\omega_{\epsilon}-\omega_{\epsilon^{\prime}}\right)\right) \mathscr{F}_{\epsilon^{\prime}} u_{\epsilon^{\prime}}^{r} \mathscr{F}_{\epsilon^{\prime}} \partial_{i}\left(\Omega_{\epsilon}-\Omega_{\epsilon^{\prime}}\right),
\end{aligned}
$$

$A_{5}$

$$
\begin{aligned}
= & \left(\left(\mathscr{F}_{\epsilon}-\mathscr{F}_{\epsilon^{\prime}}\right) \partial_{i}\left(\omega_{\epsilon}-\omega_{\epsilon^{\prime}}\right)\right) \mathscr{F}_{\epsilon} \partial_{i} B_{\epsilon} \mathscr{F}_{\epsilon} \partial_{z} \Pi_{\epsilon} \\
& +\left(\mathscr{F}_{\epsilon^{\prime}} \partial_{i}\left(\omega_{\epsilon}-\omega_{\epsilon^{\prime}}\right)\right)\left(\mathscr{F}_{\epsilon}-\mathscr{F}_{\epsilon^{\prime}}\right) \partial_{i} B_{\epsilon} \mathscr{F}_{\epsilon} \partial_{z} \Pi_{\epsilon} \\
& +\left(\mathscr{F}_{\epsilon^{\prime}} \partial_{i}\left(\omega_{\epsilon}-\omega_{\epsilon^{\prime}}\right)\right) \mathscr{F}_{\epsilon^{\prime}} \partial_{i}\left(B_{\epsilon}-B_{\epsilon^{\prime}}\right) \mathscr{F}_{\epsilon} \partial_{z} \Pi_{\epsilon} \\
& +\left(\mathscr{F}_{\epsilon^{\prime}} \partial_{i}\left(\omega_{\epsilon}-\omega_{\epsilon^{\prime}}\right)\right) \mathscr{F}_{\epsilon^{\prime}} \partial_{i} B_{\epsilon^{\prime}}\left(\mathscr{F}_{\epsilon}-\mathscr{F}_{\epsilon^{\prime}}\right) \partial_{z} \Pi_{\epsilon} \\
& +\left(\mathscr{F}_{\epsilon^{\prime}} \partial_{i}\left(\omega_{\epsilon}-\omega_{\epsilon^{\prime}}\right)\right) \mathscr{F}_{\epsilon^{\prime}} \partial_{i} B_{\epsilon^{\prime}} \mathscr{F}_{\epsilon^{\prime}} \partial_{z}\left(\Pi_{\epsilon}-\Pi_{\epsilon^{\prime}}\right),
\end{aligned}
$$


$A_{6}$

$=\left(\left(\mathscr{F}_{\epsilon}-\mathscr{F}_{\epsilon^{\prime}}\right) \partial_{i}\left(\omega_{\epsilon}-\omega_{\epsilon^{\prime}}\right)\right) \mathscr{F}_{\epsilon} B_{\epsilon} \mathscr{F}_{\epsilon} \partial_{i} \partial_{z} \Pi_{\epsilon}$

$+\left(\mathscr{F}_{\epsilon^{\prime}} \partial_{i}\left(\omega_{\epsilon}-\omega_{\epsilon^{\prime}}\right)\right)\left(\mathscr{F}_{\epsilon}-\mathscr{F}_{\epsilon^{\prime}}\right) B_{\epsilon} \mathscr{F}_{\epsilon} \partial_{i} \partial_{z} \Pi_{\epsilon}$

$+\left(\mathscr{F}_{\epsilon^{\prime}} \partial_{i}\left(\omega_{\epsilon}-\omega_{\epsilon^{\prime}}\right)\right) \mathscr{F}_{\epsilon^{\prime}}\left(B_{\epsilon}-B_{\epsilon^{\prime}}\right) \mathscr{F}_{\epsilon} \partial_{i} \partial_{z} \Pi_{\epsilon}$

$+\left(\mathscr{F}_{\epsilon^{\prime}} \partial_{i}\left(\omega_{\epsilon}-\omega_{\epsilon^{\prime}}\right)\right) \mathscr{F}_{\epsilon^{\prime}} B_{\epsilon^{\prime}}\left(\mathscr{F}_{\epsilon}-\mathscr{F}_{\epsilon^{\prime}}\right) \partial_{i} \partial_{z} \Pi_{\epsilon}$

$+\left(\mathscr{F}_{\epsilon^{\prime}} \partial_{i}\left(\omega_{\epsilon}-\omega_{\epsilon^{\prime}}\right)\right) \mathscr{F}_{\epsilon^{\prime}} B_{\epsilon^{\prime}} \mathscr{J}_{\epsilon^{\prime}} \partial_{i} \partial_{z}\left(\Pi_{\epsilon}-\Pi_{\epsilon^{\prime}}\right)$.

$A_{1}, \ldots, A_{6}$ can be estimated as follows:

$$
\begin{aligned}
\int_{\mathbb{R}^{3}} \mid & A_{1} \mid d x \\
\leq & \left(\epsilon+\epsilon^{\prime}\right)\left\|\nabla\left(\omega_{\epsilon}-\omega_{\epsilon^{\prime}}\right)\right\|_{L^{2}}\left\|\left(\mathcal{F}_{\epsilon} \partial_{i} \mathbf{u}_{\epsilon} \cdot \nabla\right) \mathcal{F}_{\epsilon} \omega_{\epsilon}\right\|_{1} \\
& +\left\|\nabla\left(\omega_{\epsilon}-\omega_{\epsilon^{\prime}}\right)\right\|_{L^{2}}\left(\epsilon+\epsilon^{\prime}\right)\left\|\nabla \mathbf{u}_{\epsilon}\right\|_{1}\left\|\nabla \omega_{\epsilon}\right\|_{L^{\infty}} \\
& +\left\|\nabla\left(\omega_{\epsilon}-\omega_{\epsilon^{\prime}}\right)\right\|_{L^{2}}^{2}\left\|\nabla \omega_{\epsilon}\right\|_{L^{\infty}} \\
& +\left\|\nabla\left(\omega_{\epsilon}-\omega_{\epsilon^{\prime}}\right)\right\|_{L^{2}}\left\|\nabla \mathbf{u}_{\epsilon^{\prime}}\right\|_{L^{\infty}}\left(\epsilon+\epsilon^{\prime}\right)\left\|\nabla \omega_{\epsilon}\right\|_{1} \\
& +\left\|\nabla\left(\omega_{\epsilon}-\omega_{\epsilon^{\prime}}\right)\right\|_{L^{2}}^{2}\left\|\nabla \mathbf{u}_{\epsilon^{\prime}}\right\|_{L^{\infty}}, \\
\int_{\mathbb{R}^{3}} \mid & A_{2} \mid d x \\
\leq & \left(\epsilon+\epsilon^{\prime}\right)\left\|\nabla\left(\omega_{\epsilon}-\omega_{\epsilon^{\prime}}\right)\right\|_{L^{2}}\left\|\left(\mathcal{F}_{\epsilon} \mathbf{u}_{\epsilon} \cdot \nabla\right) \mathcal{F}_{\epsilon} \partial_{i} \omega_{\epsilon}\right\|_{1} \\
& +\left\|\nabla\left(\omega_{\epsilon}-\omega_{\epsilon^{\prime}}\right)\right\|_{L^{2}}\left(\epsilon+\epsilon^{\prime}\right)\left\|\nabla \mathbf{u}_{\epsilon}\right\|_{L^{2}}\left\|\nabla^{2} \omega_{\epsilon}\right\|_{L^{\infty}} \\
& +2\left\|\omega_{\epsilon}-\omega_{\epsilon^{\prime}}\right\|_{1}^{2}\left\|\nabla^{2} \omega_{\epsilon}\right\|_{L^{2}} \\
& +\left\|\nabla\left(\omega_{\epsilon}-\omega_{\epsilon^{\prime}}\right)\right\|_{L^{2}}\left\|\mathbf{u}_{\epsilon^{\prime}}\right\|_{L^{\infty}}\left(\epsilon+\epsilon^{\prime}\right)\left\|\nabla^{2} \omega_{\epsilon}\right\|_{1},
\end{aligned}
$$$$
\int_{\mathbb{R}^{3}}\left|A_{3}\right| d x
$$$$
\leq\left(\epsilon+\epsilon^{\prime}\right)\left\|\nabla\left(\omega_{\epsilon}-\omega_{\epsilon^{\prime}}\right)\right\|_{L^{2}}\left\|\mathscr{F}_{\epsilon} \partial_{i} u_{\epsilon}^{r} \mathscr{F}_{\epsilon} \Omega_{\epsilon}\right\|_{1}
$$$$
+\left\|\nabla\left(\omega_{\epsilon}-\omega_{\epsilon^{\prime}}\right)\right\|_{L^{2}}\left(\epsilon+\epsilon^{\prime}\right)\left\|\nabla \mathbf{u}_{\epsilon}\right\|_{1}\left\|\Omega_{\epsilon}\right\|_{L^{\infty}}
$$$$
+\left\|\nabla\left(\omega_{\epsilon}-\omega_{\epsilon^{\prime}}\right)\right\|_{L^{2}}^{2}\left\|\Omega_{\epsilon}\right\|_{L^{\infty}}
$$$$
+\left\|\nabla\left(\omega_{\epsilon}-\omega_{\epsilon^{\prime}}\right)\right\|_{L^{2}}\left\|\nabla \mathbf{u}_{\epsilon^{\prime}}\right\|_{L^{\infty}}\left(\epsilon+\epsilon^{\prime}\right)\left\|\Omega_{\epsilon}\right\|_{1}
$$$$
+\left\|\nabla\left(\omega_{\epsilon}-\omega_{\epsilon^{\prime}}\right)\right\|_{L^{2}}\left\|\nabla \mathbf{u}_{\epsilon^{\prime}}\right\|_{L^{\infty}}\left\|\Omega_{\epsilon}-\Omega_{\epsilon^{\prime}}\right\|_{L^{2}},
$$

$$
\begin{aligned}
\int_{\mathbb{R}^{3}} & A_{4} \mid d x \\
\leq & \left(\epsilon+\epsilon^{\prime}\right)\left\|\nabla\left(\omega_{\epsilon}-\omega_{\epsilon^{\prime}}\right)\right\|_{L^{2}}\left\|\mathcal{F}_{\epsilon} u_{\epsilon}^{r} \mathcal{F}_{\epsilon} \partial_{i} \Omega_{\epsilon}\right\|_{1} \\
& +\left\|\nabla\left(\omega_{\epsilon}-\omega_{\epsilon^{\prime}}\right)\right\|_{L^{2}}\left(\epsilon+\epsilon^{\prime}\right)\left\|\nabla \mathbf{u}_{\epsilon}\right\|_{L^{2}}\left\|\nabla \Omega_{\epsilon}\right\|_{L^{\infty}} \\
& +\left\|\omega_{\epsilon}-\omega_{\epsilon^{\prime}}\right\|_{1}^{2}\left\|\nabla \Omega_{\epsilon}\right\|_{L^{2}}
\end{aligned}
$$

$+\left\|\nabla\left(\omega_{\epsilon}-\omega_{\epsilon^{\prime}}\right)\right\|_{L^{2}}\left\|\mathbf{u}_{\epsilon^{\prime}}\right\|_{L^{\infty}}\left(\epsilon+\epsilon^{\prime}\right)\left\|\nabla \Omega_{\epsilon}\right\|_{1}$

$+\left\|\nabla\left(\omega_{\epsilon}-\omega_{\epsilon^{\prime}}\right)\right\|_{L^{2}}\left\|\mathbf{u}_{\epsilon^{\prime}}\right\|_{L^{\infty}}\left\|\nabla\left(\Omega_{\epsilon}-\Omega_{\epsilon^{\prime}}\right)\right\|_{L^{2}}$,

$$
\begin{aligned}
& \int_{\mathbb{R}^{3}}\left|A_{5}\right| d x \\
& \leq\left(\epsilon+\epsilon^{\prime}\right)\left\|\nabla\left(\omega_{\epsilon}-\omega_{\epsilon^{\prime}}\right)\right\|_{L^{2}}\left\|\mathcal{F}_{\epsilon} \partial_{i} B_{\epsilon} \mathcal{g}_{\epsilon} \partial_{z} \Pi_{\epsilon}\right\|_{1} \\
&+\left\|\nabla\left(\omega_{\epsilon}-\omega_{\epsilon^{\prime}}\right)\right\|_{L^{2}}\left(\epsilon+\epsilon^{\prime}\right)\left\|\nabla B_{\epsilon}\right\|_{1}\left\|\nabla \Pi_{\epsilon}\right\|_{L^{\infty}} \\
&+\left\|\nabla\left(\omega_{\epsilon}-\omega_{\epsilon^{\prime}}\right)\right\|_{L^{2}}\left\|\nabla\left(B_{\epsilon}-B_{\epsilon^{\prime}}\right)\right\|_{L^{2}}\left\|\nabla \Pi_{\epsilon}\right\|_{L^{\infty}} \\
&+\left\|\nabla\left(\omega_{\epsilon}-\omega_{\epsilon^{\prime}}\right)\right\|_{L^{2}}\left\|\nabla B_{\epsilon^{\prime}}\right\|_{L^{\infty}}\left(\epsilon+\epsilon^{\prime}\right)\left\|\nabla \Pi_{\epsilon}\right\|_{1} \\
&+\left\|\nabla\left(\omega_{\epsilon}-\omega_{\epsilon^{\prime}}\right)\right\|_{L^{2}}\left\|\nabla B_{\epsilon^{\prime}}\right\|_{L^{\infty}}\left\|\nabla\left(\Pi_{\epsilon}-\Pi_{\epsilon^{\prime}}\right)\right\|_{L^{2}}, \\
& \int_{\mathbb{R}^{3}}\left|A_{6}\right| d x\left(\epsilon+\epsilon^{\prime}\right)\left\|\nabla\left(\omega_{\epsilon}-\omega_{\epsilon^{\prime}}\right)\right\|_{L^{2}}\left\|\mathscr{J}_{\epsilon} B_{\epsilon} \mathcal{F}_{\epsilon} \partial_{i} \partial_{z} \Pi_{\epsilon}\right\|_{1} \\
&+\left\|\nabla\left(\omega_{\epsilon}-\omega_{\epsilon^{\prime}}\right)\right\|_{L^{2}}\left(\epsilon+\epsilon^{\prime}\right)\left\|B_{\epsilon}\right\|_{1}\left\|\nabla^{2} \Pi_{\epsilon}\right\|_{L^{\infty}} \\
&+\left\|\nabla\left(\omega_{\epsilon}-\omega_{\epsilon^{\prime}}\right)\right\|_{L^{2}}\left\|B_{\epsilon}-B_{\epsilon^{\prime}}\right\|_{L^{2}}\left\|\nabla^{2} \Pi_{\epsilon}\right\|_{L^{\infty}} \\
&+\left\|\nabla\left(\omega_{\epsilon}-\omega_{\epsilon^{\prime}}\right)\right\|_{L^{2}}\left\|B_{\epsilon^{\prime}}\right\|_{L^{\infty}}\left(\epsilon+\epsilon^{\prime}\right)\left\|\nabla^{2} \Pi_{\epsilon}\right\|_{1} \\
&+\left\|\nabla\left(\omega_{\epsilon}-\omega_{\epsilon^{\prime}}\right)\right\|_{L^{2}}\left\|B_{\epsilon^{\prime}}\right\|_{L^{\infty}}\left\|\nabla^{2}\left(\Pi_{\epsilon}-\Pi_{\epsilon^{\prime}}\right)\right\|_{L^{2}} .
\end{aligned}
$$

Similarly, we can obtain the estimates for $\Pi$.

$$
\begin{aligned}
& \frac{1}{2} \frac{d}{d t}\left|\partial_{i} \partial_{j}\left(\Pi_{\epsilon}-\Pi_{\epsilon^{\prime}}\right)\right|^{2} \\
& \quad=\partial_{i} \partial_{j}\left(F_{\epsilon}^{3}\left(\omega_{\epsilon}, \Pi_{\epsilon}\right)-F_{\epsilon^{\prime}}^{3}\left(\omega_{\epsilon^{\prime}}, \Pi_{\epsilon^{\prime}}\right)\right) \partial_{i} \partial_{j}\left(\Pi_{\epsilon}-\Pi_{\epsilon^{\prime}}\right) \\
& \quad=B_{1}+B_{2}+B_{3}+B_{4}+B_{5}+B_{6}
\end{aligned}
$$

where

$$
\begin{aligned}
& B_{1}=\left(\left(\mathscr{F}_{\epsilon}-\mathscr{F}_{\epsilon^{\prime}}\right) \partial_{i} \partial_{j}\left(\Pi_{\epsilon}-\Pi_{\epsilon^{\prime}}\right)\right)\left(\mathscr{F}_{\epsilon} \partial_{i} \partial_{j} \mathbf{u}_{\epsilon} \cdot \nabla\right) \\
& \text { - } \mathscr{F}_{\epsilon} \Pi_{\epsilon}+\left(\mathscr{F}_{\epsilon^{\prime}} \partial_{i} \partial_{j}\left(\Pi_{\epsilon}-\Pi_{\epsilon^{\prime}}\right)\right) \\
& \cdot\left(\left(\mathscr{F}_{\epsilon}-\mathscr{J}_{\epsilon^{\prime}}\right) \partial_{i} \partial_{j} \mathbf{u}_{\epsilon} \cdot \nabla\right) \mathscr{F}_{\epsilon} \Pi_{\epsilon} \\
& +\left(\mathscr{F}_{\epsilon^{\prime}} \partial_{i} \partial_{j}\left(\Pi_{\epsilon}-\Pi_{\epsilon^{\prime}}\right)\right)\left(\mathscr{F}_{\epsilon^{\prime}} \partial_{i} \partial_{j}\left(\mathbf{u}_{\epsilon}-\mathbf{u}_{\epsilon^{\prime}}\right) \cdot \nabla\right) \\
& \cdot \mathscr{J}_{\epsilon} \Pi_{\epsilon}+\left(\mathscr{F}_{\epsilon^{\prime}} \partial_{i} \partial_{j}\left(\Pi_{\epsilon}-\Pi_{\epsilon^{\prime}}\right)\right)\left(\mathscr{F}_{\epsilon^{\prime}} \partial_{i} \partial_{j} \mathbf{u}_{\epsilon^{\prime}} \cdot \nabla\right) \\
& \cdot\left(\mathscr{F}_{\epsilon}-\mathscr{F}_{\epsilon^{\prime}}\right) \Pi_{\epsilon}+\left(\mathscr{F}_{\epsilon^{\prime}} \partial_{i} \partial_{j}\left(\Pi_{\epsilon}-\Pi_{\epsilon^{\prime}}\right)\right) \\
& \cdot\left(\mathscr{F}_{\epsilon^{\prime}} \partial_{i} \partial_{j} \mathbf{u}_{\epsilon^{\prime}} \cdot \nabla\right) \mathscr{F}_{\epsilon^{\prime}}\left(\Pi_{\epsilon}-\Pi_{\epsilon^{\prime}}\right) \text {, } \\
& B_{2}=\left(\left(\mathscr{F}_{\epsilon}-\mathscr{F}_{\epsilon^{\prime}}\right) \partial_{i} \partial_{j}\left(\Pi_{\epsilon}-\Pi_{\epsilon^{\prime}}\right)\right)\left(\mathscr{F}_{\epsilon} \partial_{i} \mathbf{u}_{\epsilon} \cdot \nabla\right) \\
& \text { - } \mathscr{J}_{\epsilon} \partial_{j} \Pi_{\epsilon}+\left(\mathscr{F}_{\epsilon^{\prime}} \partial_{i} \partial_{j}\left(\Pi_{\epsilon}-\Pi_{\epsilon^{\prime}}\right)\right) \\
& \cdot\left(\left(\mathscr{F}_{\epsilon}-\mathscr{J}_{\epsilon^{\prime}}\right) \partial_{i} \mathbf{u}_{\epsilon} \cdot \nabla\right) \mathscr{F}_{\epsilon} \partial_{j} \Pi_{\epsilon}
\end{aligned}
$$




$$
\begin{aligned}
& +\left(\mathscr{F}_{\epsilon^{\prime}} \partial_{i} \partial_{j}\left(\Pi_{\epsilon}-\Pi_{\epsilon^{\prime}}\right)\right)\left(\mathscr{F}_{\epsilon^{\prime}} \partial_{i}\left(\mathbf{u}_{\epsilon}-\mathbf{u}_{\epsilon^{\prime}}\right) \cdot \nabla\right) \\
& \cdot \mathscr{J}_{\epsilon} \partial_{j} \Pi_{\epsilon}+\left(\mathscr{F}_{\epsilon^{\prime}} \partial_{i} \partial_{j}\left(\Pi_{\epsilon}-\Pi_{\epsilon^{\prime}}\right)\right)\left(\mathscr{F}_{\epsilon^{\prime}} \partial_{i} \mathbf{u}_{\epsilon^{\prime}} \cdot \nabla\right) \\
& \cdot\left(\mathscr{F}_{\epsilon}-\mathscr{F}_{\epsilon^{\prime}}\right) \partial_{j} \Pi_{\epsilon}+\left(\mathscr{F}_{\epsilon^{\prime}} \partial_{i} \partial_{j}\left(\Pi_{\epsilon}-\Pi_{\epsilon^{\prime}}\right)\right) \\
& \cdot\left(\mathscr{F}_{\epsilon^{\prime}} \partial_{i} \mathbf{u}_{\epsilon^{\prime}} \cdot \nabla\right) \mathscr{F}_{\epsilon^{\prime}} \partial_{j}\left(\Pi_{\epsilon}-\Pi_{\epsilon^{\prime}}\right) \text {, } \\
& B_{3}=\left(\left(\mathscr{F}_{\epsilon}-\mathscr{F}_{\epsilon^{\prime}}\right) \partial_{i} \partial_{j}\left(\Pi_{\epsilon}-\Pi_{\epsilon^{\prime}}\right)\right)\left(\mathscr{F}_{\epsilon} \mathbf{u}_{\epsilon} \cdot \nabla\right) \\
& \cdot \mathscr{J}_{\epsilon} \partial_{i} \partial_{j} \Pi_{\epsilon}+\left(\mathscr{F}_{\epsilon^{\prime}} \partial_{i} \partial_{j}\left(\Pi_{\epsilon}-\Pi_{\epsilon^{\prime}}\right)\right) \\
& \cdot\left(\left(\mathscr{F}_{\epsilon}-\mathscr{F}_{\epsilon^{\prime}}\right) \mathbf{u}_{\epsilon} \cdot \nabla\right) \mathscr{F}_{\epsilon} \partial_{i} \partial_{j} \Pi_{\epsilon} \\
& +\left(\mathscr{F}_{\epsilon^{\prime}} \partial_{i} \partial_{j}\left(\Pi_{\epsilon}-\Pi_{\epsilon^{\prime}}\right)\right)\left(\mathscr{F}_{\epsilon^{\prime}}\left(\mathbf{u}_{\epsilon}-\mathbf{u}_{\epsilon^{\prime}}\right) \cdot \nabla\right) \\
& \cdot \mathscr{J}_{\epsilon} \partial_{i} \partial_{j} \Pi_{\epsilon}+\left(\mathscr{F}_{\epsilon^{\prime}} \partial_{i} \partial_{j}\left(\Pi_{\epsilon}-\Pi_{\epsilon^{\prime}}\right)\right)\left(\mathscr{F}_{\epsilon^{\prime}} \mathbf{u}_{\epsilon^{\prime}} \cdot \nabla\right) \\
& \cdot\left(\mathscr{F}_{\epsilon}-\mathscr{F}_{\epsilon^{\prime}}\right) \partial_{i} \partial_{j} \Pi_{\epsilon}+\left(\mathscr{F}_{\epsilon^{\prime}} \partial_{i} \partial_{j}\left(\Pi_{\epsilon}-\Pi_{\epsilon^{\prime}}\right)\right) \\
& \cdot\left(\mathscr{F}_{\epsilon^{\prime}} \mathbf{u}_{\epsilon^{\prime}} \cdot \nabla\right) \mathscr{F}_{\epsilon^{\prime}} \partial_{i} \partial_{j}\left(\Pi_{\epsilon}-\Pi_{\epsilon^{\prime}}\right) \text {, } \\
& B_{4}=\left(\left(\mathscr{F}_{\epsilon}-\mathscr{F}_{\epsilon^{\prime}}\right) \partial_{i} \partial_{j}\left(\Pi_{\epsilon}-\Pi_{\epsilon^{\prime}}\right)\right) \mathscr{F}_{\epsilon} \partial_{i} \partial_{j} \Pi_{\epsilon} \mathscr{F}_{\epsilon} \partial_{z} \Pi_{\epsilon} \\
& +\left(\mathscr{F}_{\epsilon^{\prime}} \partial_{i} \partial_{j}\left(\Pi_{\epsilon}-\Pi_{\epsilon^{\prime}}\right)\right)\left(\mathscr{F}_{\epsilon}-\mathscr{F}_{\epsilon^{\prime}}\right) \partial_{i} \partial_{j} \Pi_{\epsilon} \mathscr{F}_{\epsilon} \partial_{z} \Pi_{\epsilon} \\
& +\left(\mathscr{F}_{\epsilon^{\prime}} \partial_{i} \partial_{j}\left(\Pi_{\epsilon}-\Pi_{\epsilon^{\prime}}\right)\right) \mathscr{F}_{\epsilon^{\prime}} \partial_{i} \partial_{j}\left(\Pi_{\epsilon}-\Pi_{\epsilon^{\prime}}\right) \mathscr{F}_{\epsilon} \partial_{z} \Pi_{\epsilon} \\
& +\left(\mathscr{F}_{\epsilon^{\prime}} \partial_{i} \partial_{j}\left(\Pi_{\epsilon}-\Pi_{\epsilon^{\prime}}\right)\right) \mathscr{F}_{\epsilon^{\prime}} \partial_{i} \partial_{j} \Pi_{\epsilon^{\prime}}\left(\mathscr{F}_{\epsilon}-\mathscr{F}_{\epsilon^{\prime}}\right) \partial_{z} \Pi_{\epsilon} \\
& +\left(\mathscr{F}_{\epsilon^{\prime}} \partial_{i} \partial_{j}\left(\Pi_{\epsilon}-\Pi_{\epsilon^{\prime}}\right)\right) \\
& \text { - } \mathscr{F}_{\epsilon^{\prime}} \partial_{i} \partial_{j} \Pi_{\epsilon^{\prime}} \mathscr{F}_{\epsilon^{\prime}} \partial_{z}\left(\Pi_{\epsilon}-\Pi_{\epsilon^{\prime}}\right) \text {, } \\
& B_{5}=\left(\left(\mathscr{F}_{\epsilon}-\mathscr{J}_{\epsilon^{\prime}}\right) \partial_{i} \partial_{j}\left(\Pi_{\epsilon}-\Pi_{\epsilon^{\prime}}\right)\right) \mathscr{F}_{\epsilon} \partial_{i} \Pi_{\epsilon} \mathscr{F}_{\epsilon} \partial_{j} \partial_{z} \Pi_{\epsilon} \\
& +\left(\mathscr{F}_{\epsilon^{\prime}} \partial_{i} \partial_{j}\left(\Pi_{\epsilon}-\Pi_{\epsilon^{\prime}}\right)\right)\left(\mathscr{F}_{\epsilon}-\mathscr{F}_{\epsilon^{\prime}}\right) \partial_{i} \Pi_{\epsilon} \mathscr{F}_{\epsilon} \partial_{j} \partial_{z} \Pi_{\epsilon} \\
& +\left(\mathscr{F}_{\epsilon^{\prime}} \partial_{i} \partial_{j}\left(\Pi_{\epsilon}-\Pi_{\epsilon^{\prime}}\right)\right) \mathscr{F}_{\epsilon^{\prime}} \partial_{i}\left(\Pi_{\epsilon}-\Pi_{\epsilon^{\prime}}\right) \mathscr{F}_{\epsilon} \partial_{j} \partial_{z} \Pi_{\epsilon} \\
& +\left(\mathscr{F}_{\epsilon^{\prime}} \partial_{i} \partial_{j}\left(\Pi_{\epsilon}-\Pi_{\epsilon^{\prime}}\right)\right) \mathscr{F}_{\epsilon^{\prime}} \partial_{i} \Pi_{\epsilon^{\prime}}\left(\mathscr{F}_{\epsilon}-\mathscr{F}_{\epsilon^{\prime}}\right) \partial_{j} \partial_{z} \Pi_{\epsilon} \\
& +\left(\mathscr{F}_{\epsilon^{\prime}} \partial_{i} \partial_{j}\left(\Pi_{\epsilon}-\Pi_{\epsilon^{\prime}}\right)\right) \\
& \text { - } \mathscr{F}_{\epsilon^{\prime}} \partial_{i} \Pi_{\epsilon^{\prime}} \mathscr{F}_{\epsilon^{\prime}} \partial_{j} \partial_{z}\left(\Pi_{\epsilon}-\Pi_{\epsilon^{\prime}}\right) \text {, } \\
& B_{6}=\left(\left(\mathscr{F}_{\epsilon}-\mathscr{F}_{\epsilon^{\prime}}\right) \partial_{i} \partial_{j}\left(\Pi_{\epsilon}-\Pi_{\epsilon^{\prime}}\right)\right) \mathscr{F}_{\epsilon} \Pi_{\epsilon} \mathscr{F}_{\epsilon} \partial_{i} \partial_{j} \partial_{z} \Pi_{\epsilon} \\
& +\left(\mathscr{F}_{\epsilon^{\prime}} \partial_{i} \partial_{j}\left(\Pi_{\epsilon}-\Pi_{\epsilon^{\prime}}\right)\right)\left(\mathscr{F}_{\epsilon}-\mathscr{F}_{\epsilon^{\prime}}\right) \Pi_{\epsilon} \mathscr{F}_{\epsilon} \partial_{i} \partial_{j} \partial_{z} \Pi_{\epsilon} \\
& +\left(\mathscr{F}_{\epsilon^{\prime}} \partial_{i} \partial_{j}\left(\Pi_{\epsilon}-\Pi_{\epsilon^{\prime}}\right)\right) \mathscr{F}_{\epsilon^{\prime}}\left(\Pi_{\epsilon}-\Pi_{\epsilon^{\prime}}\right) \mathscr{F}_{\epsilon} \partial_{i} \partial_{j} \partial_{z} \Pi_{\epsilon} \\
& +\left(\mathscr{F}_{\epsilon^{\prime}} \partial_{i} \partial_{j}\left(\Pi_{\epsilon}-\Pi_{\epsilon^{\prime}}\right)\right) \mathscr{F}_{\epsilon^{\prime}} \Pi_{\epsilon^{\prime}}\left(\mathscr{F}_{\epsilon}-\mathscr{F}_{\epsilon^{\prime}}\right) \partial_{i} \partial_{j} \partial_{z} \Pi_{\epsilon} \\
& +\left(\mathscr{F}_{\epsilon^{\prime}} \partial_{i} \partial_{j}\left(\Pi_{\epsilon}-\Pi_{\epsilon^{\prime}}\right)\right) \\
& \text { - } \mathscr{F}_{\epsilon^{\prime}} \partial_{i} \Pi_{\epsilon^{\prime}} \mathscr{F}_{\epsilon^{\prime}} \partial_{i} \partial_{j} \partial_{z}\left(\Pi_{\epsilon}-\Pi_{\epsilon^{\prime}}\right) \text {. }
\end{aligned}
$$

These $B_{1}, \ldots, B_{6}$ can be estimated similarly.

$$
\begin{aligned}
& \int_{\mathbb{R}^{3}}\left|B_{1}\right| d x \\
& \leq\left(\epsilon+\epsilon^{\prime}\right)\left\|\nabla^{2}\left(\Pi_{\epsilon}-\Pi_{\epsilon^{\prime}}\right)\right\|_{L^{2}}\left\|\left(\mathcal{F}_{\epsilon} \partial_{i} \partial_{j} \mathbf{u}_{\epsilon} \cdot \nabla\right) \mathscr{J}_{\epsilon} \Pi_{\epsilon}\right\|_{1} \\
& \quad+\left\|\nabla^{2}\left(\Pi_{\epsilon}-\Pi_{\epsilon^{\prime}}\right)\right\|_{L^{2}}\left(\epsilon+\epsilon^{\prime}\right)\left\|\nabla^{2} \mathbf{u}_{\epsilon}\right\|_{1}\left\|\nabla \Pi_{\epsilon}\right\|_{L^{\infty}} \\
& \quad+\left\|\nabla^{2}\left(\Pi_{\epsilon}-\Pi_{\epsilon^{\prime}}\right)\right\|_{L^{2}}\left\|\nabla\left(\omega_{\epsilon}-\omega_{\epsilon^{\prime}}\right)\right\|_{L^{2}}\left\|\nabla \Pi_{\epsilon}\right\|_{L^{\infty}} \\
& \quad+\left\|\nabla^{2}\left(\Pi_{\epsilon}-\Pi_{\epsilon^{\prime}}\right)\right\|_{L^{2}}\left\|\nabla^{2} \mathbf{u}_{\epsilon^{\prime}}\right\|_{L^{\infty}}\left(\epsilon+\epsilon^{\prime}\right)\left\|\nabla \Pi_{\epsilon}\right\|_{1} \\
& \quad+\left\|\Pi_{\epsilon}-\Pi_{\epsilon^{\prime}}\right\|_{2}^{2}\left\|\nabla^{2} \mathbf{u}_{\epsilon^{\prime}}\right\|_{L^{\infty}}, \\
& \int_{\mathbb{R}^{3}}\left|B_{2}\right| d x \\
& \leq\left(\epsilon+\epsilon^{\prime}\right)\left\|\nabla^{2}\left(\Pi_{\epsilon}-\Pi_{\epsilon^{\prime}}\right)\right\|_{L^{2}}\left\|\left(\mathscr{J}_{\epsilon} \partial_{i} \mathbf{u}_{\epsilon} \cdot \nabla\right) \mathscr{F}_{\epsilon} \partial_{j} \Pi_{\epsilon}\right\|_{1} \\
& \quad+\left\|\nabla^{2}\left(\Pi_{\epsilon}-\Pi_{\epsilon^{\prime}}\right)\right\|_{L^{2}}\left(\epsilon+\epsilon^{\prime}\right)\left\|\nabla \mathbf{u}_{\epsilon}\right\|_{1}\left\|\nabla^{2} \Pi_{\epsilon}\right\|_{L^{\infty}} \\
& \quad+\left\|\nabla^{2}\left(\Pi_{\epsilon}-\Pi_{\epsilon^{\prime}}\right)\right\|_{L^{2}}\left\|\omega_{\epsilon}-\omega_{\epsilon^{\prime}}\right\|_{L^{2}}\left\|\nabla^{2} \Pi_{\epsilon}\right\|_{L^{\infty}} \\
& \quad+\left\|\nabla^{2}\left(\Pi_{\epsilon}-\Pi_{\epsilon^{\prime}}\right)\right\|_{L^{2}}\left\|\nabla \mathbf{u}_{\epsilon^{\prime}}\right\|_{L^{\infty}}\left(\epsilon+\epsilon^{\prime}\right)\left\|\nabla^{2} \Pi_{\epsilon}\right\|_{1} \\
& \quad+\left\|\nabla^{2}\left(\Pi_{\epsilon}-\Pi_{\epsilon^{\prime}}\right)\right\|_{L^{2}}^{2}\left\|\nabla \mathbf{u}_{\epsilon^{\prime}}\right\|_{L^{\infty}}, \\
& \int_{\mathbb{R}^{3}}\left|B_{3}\right| d x
\end{aligned}
$$
$\leq\left(\epsilon+\epsilon^{\prime}\right)\left\|\nabla^{2}\left(\Pi_{\epsilon}-\Pi_{\epsilon^{\prime}}\right)\right\|_{L^{2}}\left\|\left(\mathscr{F}_{\epsilon} \mathbf{u}_{\epsilon} \cdot \nabla\right) \mathscr{F}_{\epsilon} \partial_{i} \partial_{j} \Pi_{\epsilon}\right\|_{1}$$$
+\left\|\nabla^{2}\left(\Pi_{\epsilon}-\Pi_{\epsilon^{\prime}}\right)\right\|_{L^{2}}\left(\epsilon+\epsilon^{\prime}\right)\left\|\nabla \mathbf{u}_{\epsilon}\right\|_{L^{2}}\left\|\nabla^{3} \Pi_{\epsilon}\right\|_{L^{\infty}}
$$$$
+\left\|\nabla^{2}\left(\Pi_{\epsilon}-\Pi_{\epsilon^{\prime}}\right)\right\|_{L^{2}}\left\|\omega_{\epsilon}-\omega_{\epsilon^{\prime}}\right\|_{1}\left\|\nabla^{3} \Pi_{\epsilon}\right\|_{L^{2}}
$$$$
+\left\|\nabla^{2}\left(\Pi_{\epsilon}-\Pi_{\epsilon^{\prime}}\right)\right\|_{L^{2}}\left\|\mathbf{u}_{\epsilon^{\prime}}\right\|_{L^{\infty}}\left(\epsilon+\epsilon^{\prime}\right)\left\|\nabla^{3} \Pi_{\epsilon}\right\|_{1},
$$$$
\int_{\mathbb{R}^{3}}\left|B_{4}\right| d x
$$

$$
\begin{aligned}
\leq & \left(\epsilon+\epsilon^{\prime}\right)\left\|\nabla^{2}\left(\Pi_{\epsilon}-\Pi_{\epsilon^{\prime}}\right)\right\|_{L^{2}}\left\|\mathscr{F}_{\epsilon} \partial_{i} \partial_{j} \Pi_{\epsilon} \mathscr{J}_{\epsilon} \partial_{z} \Pi_{\epsilon}\right\|_{1} \\
& +\left\|\nabla^{2}\left(\Pi_{\epsilon}-\Pi_{\epsilon^{\prime}}\right)\right\|_{L^{2}}\left(\epsilon+\epsilon^{\prime}\right)\left\|\nabla^{2} \Pi_{\epsilon}\right\|_{1}\left\|\nabla \Pi_{\epsilon}\right\|_{L^{\infty}} \\
& +\left\|\nabla^{2}\left(\Pi_{\epsilon}-\Pi_{\epsilon^{\prime}}\right)\right\|_{L^{2}}^{2}\left\|\nabla \Pi_{\epsilon}\right\|_{L^{\infty}} \\
& +\left\|\nabla^{2}\left(\Pi_{\epsilon}-\Pi_{\epsilon^{\prime}}\right)\right\|_{L^{2}}\left\|\nabla^{2} \Pi_{\epsilon^{\prime}}\right\|_{L^{\infty}}\left(\epsilon+\epsilon^{\prime}\right)\left\|\nabla \Pi_{\epsilon}\right\|_{1} \\
& +\left\|\left(\Pi_{\epsilon}-\Pi_{\epsilon^{\prime}}\right)\right\|_{2}^{2}\left\|\nabla^{2} \Pi_{\epsilon^{\prime}}\right\|_{L^{\infty}}, \\
\int_{\mathbb{R}^{3}} & \left|B_{5}\right| d x \\
\leq & \left(\epsilon+\epsilon^{\prime}\right)\left\|\nabla^{2}\left(\Pi_{\epsilon}-\Pi_{\epsilon^{\prime}}\right)\right\|_{L^{2}}\left\|\mathscr{F}_{\epsilon} \partial_{i} \Pi_{\epsilon} \mathscr{F}_{\epsilon} \partial_{j} \partial_{z} \Pi_{\epsilon}\right\|_{1} \\
& +\left\|\nabla^{2}\left(\Pi_{\epsilon}-\Pi_{\epsilon^{\prime}}\right)\right\|_{L^{2}}\left(\epsilon+\epsilon^{\prime}\right)\left\|\nabla \Pi_{\epsilon}\right\|_{1}\left\|\nabla^{2} \Pi_{\epsilon}\right\|_{L^{\infty}} \\
& +\left\|\left(\Pi_{\epsilon}-\Pi_{\epsilon^{\prime}}\right)\right\|_{2}^{2}\left\|\nabla^{2} \Pi_{\epsilon}\right\|_{L^{\infty}}
\end{aligned}
$$




$$
\begin{aligned}
& +\left\|\nabla^{2}\left(\Pi_{\epsilon}-\Pi_{\epsilon^{\prime}}\right)\right\|_{L^{2}}\left\|\nabla \Pi_{\epsilon^{\prime}}\right\|_{L^{\infty}}\left(\epsilon+\epsilon^{\prime}\right)\left\|\nabla^{2} \Pi_{\epsilon}\right\|_{1} \\
& +\left\|\nabla^{2}\left(\Pi_{\epsilon}-\Pi_{\epsilon^{\prime}}\right)\right\|_{L^{2}}^{2}\left\|\nabla \Pi_{\epsilon^{\prime}}\right\|_{L^{\infty}}, \\
& \int_{\mathbb{R}^{3}}\left|B_{6}\right| d x \\
& \leq\left(\epsilon+\epsilon^{\prime}\right)\left\|\nabla^{2}\left(\Pi_{\epsilon}-\Pi_{\epsilon^{\prime}}\right)\right\|_{L^{2}}\left\|\mathscr{J}_{\epsilon} \Pi_{\epsilon} \mathcal{F}_{\epsilon} \partial_{i} \partial_{j} \partial_{z} \Pi_{\epsilon}\right\|_{1} \\
& \quad+\left\|\nabla^{2}\left(\Pi_{\epsilon}-\Pi_{\epsilon^{\prime}}\right)\right\|_{L^{2}}\left(\epsilon+\epsilon^{\prime}\right)\left\|\Pi_{\epsilon}\right\|_{1}\left\|\nabla^{3} \Pi_{\epsilon}\right\|_{L^{\infty}} \\
& \quad+\left\|\nabla^{2}\left(\Pi_{\epsilon}-\Pi_{\epsilon^{\prime}}\right)\right\|_{2}^{2}\left\|\nabla^{3} \Pi_{\epsilon}\right\|_{L^{\infty}} \\
& \quad+\left\|\nabla^{2}\left(\Pi_{\epsilon}-\Pi_{\epsilon^{\prime}}\right)\right\|_{L^{2}}\left\|\Pi_{\epsilon^{\prime}}\right\|_{L^{\infty}}\left(\epsilon+\epsilon^{\prime}\right)\left\|\nabla^{3} \Pi_{\epsilon}\right\|_{1} .
\end{aligned}
$$

The other terms $B$ and $\Pi+\Omega$ can be estimated similarly, so we omit the details. Then we have

$$
\begin{aligned}
& \frac{1}{2} \frac{d}{d t}\|S\|_{X^{1}}(t):=\frac{1}{2} \frac{d}{d t}\left(\left\|\omega_{\epsilon}-\omega_{\epsilon^{\prime}}\right\|_{1}+\left\|B_{\epsilon}-B_{\epsilon^{\prime}}\right\|_{1}\right. \\
& \left.\quad+\left\|\Pi_{\epsilon}-\Pi_{\epsilon^{\prime}}\right\|_{2}+\left\|\left(\Pi_{\epsilon}+\Omega_{\epsilon}\right)-\left(\Pi_{\epsilon^{\prime}}+\Omega_{\epsilon^{\prime}}\right)\right\|_{1}\right) \\
& \leq\left(\left(\epsilon+\epsilon^{\prime}\right)+\|S\|_{X^{1}}(t)\right)\left(\left\|\nabla^{3} \Pi_{\epsilon}\right\|_{L^{\infty}}+\left\|\nabla^{2} \omega_{\epsilon}\right\|_{L^{\infty}}\right. \\
& \left.\quad+\left\|\nabla^{2} B_{\epsilon}\right\|_{L^{\infty}}+\left\|\nabla^{2}\left(\Pi_{\epsilon}+\Omega_{\epsilon}\right)\right\|_{L^{\infty}}\right) \leq C\left(\left(\epsilon+\epsilon^{\prime}\right)\right. \\
& \left.\quad+\|S\|_{X^{1}}(t)\right),
\end{aligned}
$$

for $m>7 / 2$. Gronwall's inequality gives us

$$
\sup _{0 \leq t \leq T_{0}}\|S\|_{X^{1}}(t) \leq\left(\epsilon+\epsilon^{\prime}\right) e^{C T_{0}},
$$

which implies that $\left\{S_{\epsilon}\right\} \in C\left(\left[0, T_{0}\right], X^{1}\right)$ and this information completes the proof.

Proof of Theorem 1. With the bounds in Proposition 7, if we use the Sobolev inequality, then we can obtain the higher order convergence, i.e., $S \in C\left(0, T_{0}\right.$; $\left.X^{s}\right)$ for all $s<m$ by the following inequality

$$
\sup _{0 \leq t \leq T_{0}}\left\|S_{\epsilon^{\prime}}-S_{\epsilon}\right\|_{X^{s}} \leq C\left\|S_{\epsilon^{\prime}}-S_{\epsilon}\right\|_{X^{1}}^{1-s / m}\left\|S_{\epsilon^{\prime}}-S_{\epsilon}\right\|_{m}^{s / m} .
$$

Now, to show $S \in C\left(0, T_{0} ; X^{m}\right) \cap \operatorname{Lip}\left(0, T_{0} ; X^{m-1}\right)$ where $S$ satisfies our equations in classical sense almost every time, we begin the process of obtaining the right continuity at $t=0$ first. Because $X^{m}$ is a reflexive Banach space, by Proposition 7, there exist a subsequence and limit functions $S(t) \in L^{\infty}\left(0, T_{0} ; H^{m}\right)$ which satisfies $\partial_{t} S(t) \in L^{\infty}\left(0, T_{0} ; H^{m-1}\right)$ for any $0 \leq t \leq T_{0}, S_{\epsilon_{n}}(t) \rightarrow S(t)$ in $X^{m}$ and $\partial_{t} S_{\epsilon_{n}}(t) \rightarrow$ $\partial_{t} S(t)$ in $X^{m-1}$. This implies that $\|S(t)\|_{X^{m}} \leq S_{0} /\left(1-C_{0} S_{0} t\right)$, $\left\|\partial_{t} S(t)\right\|_{X^{m-1}} \leq\left(S_{0} /\left(1-C_{0} S_{0} t\right)\right)^{2}$. Thus we have

$$
\limsup _{t \rightarrow 0+}\|S\|_{X^{m}}(t) \leq S_{0} .
$$

If we use the above result, $S(t) \in C\left(0, T_{0} ; X^{s}\right)$ for any $s<m$, then $S \in C_{w}\left(0, T_{0} ; X^{m}\right)$ is obtained by the following estimate.
For arbitrary $\delta>0$ and $\varphi \in X^{-m}$, there exists $\tilde{\varphi} \in X^{-s}$ such that $\|\varphi-\widetilde{\varphi}\|_{X^{-m}} \leq \delta / 8 S_{0}$,

$$
\begin{aligned}
\left|\left[\varphi, S_{\epsilon}-S\right]_{X^{m}}\right| \leq & \|\varphi-\widetilde{\varphi}\|_{X^{-m}}\left\|S_{\epsilon}-S\right\|_{X^{m}} \\
& +\left|\left[\widetilde{\varphi}, S_{\epsilon}-S\right]_{X^{s}}\right| \\
\leq & \delta+\left|\left[\widetilde{\varphi}, S_{\epsilon}-S\right]_{X^{s}}\right|,
\end{aligned}
$$

where $[\cdot, \cdot]_{X^{k}}$ is a dual pairing on $X^{-k} \times X^{k}$. If we choose $\varphi=$ $\nabla^{2 m} \omega_{0} \in H^{-m}$, then by the weak continuity,

$$
\begin{aligned}
\left|\left[\varphi, \omega_{0}\right]_{H^{m}}\right| & \leq\left|\left[\varphi, \omega(t)-\omega_{0}\right]_{H^{m}}\right|+\left|[\varphi, \omega(t)]_{H^{m}}\right| \\
& \leq \delta+\left|[\varphi, \omega(t)]_{H^{m}}\right|, \\
\left\|\nabla^{m} \omega_{0}\right\|_{L^{2}}^{2} & \leq \delta+\left\|\nabla^{m} \omega(t)\right\|_{L^{2}}\left\|\nabla^{m} \omega_{0}\right\|_{L^{2}} \\
& \leq \delta+\frac{\left\|\nabla^{m} \omega(t)\right\|_{L^{2}}^{2}}{2}+\frac{\left\|\nabla^{m} \omega_{0}\right\|_{L^{2}}^{2}}{2} .
\end{aligned}
$$

Similarly, we have

$$
\liminf _{t \longrightarrow 0+}\|S\|_{X^{m}}(t) \geq S_{0} .
$$

By inequalities (71) and (75), we have the right continuity of $S(t)$ at $t=0$. If we apply the standard time translation invariant property and the time reversal techique, we also have $S(t) \in C\left(0, T_{0} ; H^{m}\right)$ without any difficulty. Lipschitz continuity also can be obtained by

$$
\begin{aligned}
\left\|S\left(t_{1}\right)-S\left(t_{2}\right)\right\|_{X^{m-1}} \leq & \delta+\left\|S_{\epsilon}\left(t_{1}\right)-S_{\epsilon}\left(t_{2}\right)\right\|_{X^{m-1}} \\
\leq & \delta+\left\|\int_{t_{2}}^{t_{1}} \partial_{t} S_{\epsilon}(\tau) d \tau\right\|_{X^{m-1}} \\
\leq & \delta \\
& +\left|t_{1}-t_{2}\right| \sup _{0 \leq \tau \leq T_{0}}\left\|\partial_{t} S_{\epsilon}(\tau)\right\|_{X^{m-1}} \\
\leq & \delta+\left(4 S_{0}\right)^{2}\left|t_{1}-t_{2}\right|,
\end{aligned}
$$

which means $S \in C\left(0, T_{0} ; X^{m}\right) \cap \operatorname{Lip}\left(0, T_{0} ; X^{m-1}\right)$. Hence it is a classical solution to (21) almost every time.

Next we assume that the initial data $\left(\mathbf{u}_{0}, B_{0}, \Pi_{0}, \Omega_{0}\right)$ is axisymmetric. Then the axisymmetry of a classical solution to $(21)$ is preserved and $(\omega, B, \Pi, \Omega)$ is axisymmetric solution to (21). Now we go back to (9) with $\nu=\kappa=0$, and set

$$
\begin{gathered}
u_{0} \in H^{m}\left(\mathbb{R}^{3}\right), \\
\frac{\text { curl } u_{0}}{r} \in H^{m-1}\left(\mathbb{R}^{3}\right), \\
B_{0} \in H^{m}\left(\mathbb{R}^{3}\right), \\
\frac{B_{0}}{r} \in H^{m}\left(\mathbb{R}^{3}\right),
\end{gathered}
$$


Then for

$$
\begin{aligned}
& \omega_{0}=\operatorname{curl} u_{0}, \\
& \Omega_{0}=\frac{\omega_{0}}{r}, \\
& \Pi_{0}=\frac{B_{0}}{r},
\end{aligned}
$$

we know that there exists a unique solution $(\omega, B, \Omega) \in$ $C\left(0, T_{0} ; H^{m-1}\right) \cap \operatorname{Lip}\left(0, T_{0} ; H^{m-2}\right), \Pi \in C\left(0, T_{0} ; H^{m}\right) \cap$ $\operatorname{Lip}\left(0, T_{0} ; H^{m-1}\right)$. But if we replace $\Omega$ with $\omega / r$ and $\Pi$ with $B / r$, then $\omega, B, \omega / r, B / r$ also satisfy (21) with the initial data. So by the uniqueness, $\Omega=\omega / r$ in $C\left(0, T_{0} ; H^{m-1}\right) \cap \operatorname{Lip}\left(0, T_{0} ; H^{m-2}\right)$ and $\Pi=B / r$ in $C\left(0, T_{0} ; H^{m}\right) \cap \operatorname{Lip}\left(0, T_{0} ; H^{m-1}\right)$. Next, we can show that $\mathbf{u}=\Phi * \operatorname{curl} \omega \in C\left(0, T_{0} ; H^{m}\right) \cap \operatorname{Lip}\left(0, T_{0} ; H^{m-1}\right)$. By the Poincaré lemma, curlu $=\omega$ satisfies the $\omega$ equation of (9). Then we can deduce that $\mathbf{u}$ and $B$ satisfy (9) almost every time by finding the axisymmetric scalar pressure $p$. Then the energy inequality (4) implies that $u \in L^{\infty}\left(0, T_{0} ; L^{2}\right)$ and, almost every time,

$$
\begin{aligned}
& \left\|u\left(t_{1}\right)-u\left(t_{2}\right)\right\|_{L^{2}\left(\mathbb{R}^{3}\right)} \leq\left|t_{1}-t_{2}\right| \\
& \cdot \int_{t_{2}}^{t_{1}}\left\|\partial_{t} u\left(t_{1}\right)-\partial_{t} u\left(t_{2}\right)\right\|_{L^{2}\left(\mathbb{R}^{3}\right)}(t) d t \leq\left|t_{1}-t_{2}\right| \\
& \cdot \sup _{0 \leq t \leq T_{0}}\left\|\partial_{t} u\right\|_{L^{2}} \leq\left|t_{1}-t_{2}\right| \sup _{0 \leq t \leq T_{0}}\left(\|u\|_{L^{\infty}}\|\nabla u\|_{L^{2}}\right. \\
& \left.+\|(\nabla \times B) \times B\|_{L^{2}}+\|\nabla p\|_{L^{2}}\right) \leq\left|t_{1}-t_{2}\right| \\
& \quad \cdot \sup _{0 \leq t \leq T_{0}}\left(\|\omega\|_{1}^{2}+\|B\|_{2}^{2}\right)<C\left|t_{1}-t_{2}\right|
\end{aligned}
$$

which implies $\mathbf{u} \in C\left(0, T_{0} ; H^{m}\right) \cap \operatorname{Lip}\left(0, T_{0} ; H^{m-1}\right)$. The uniqueness of $\mathbf{u}$ can be obtained from the standard techniques and we omit the details. Finally we can show that $B \in C\left(0, T_{0} ; H^{m}\right) \cap \operatorname{Lip}\left(0, T_{0} ; H^{m-1}\right)$. Almost every time, we can rewrite the $B$ equation with

$$
\partial_{t} B+\left(u^{r} \partial_{r}+u^{z} \partial_{z}\right) B=u^{r} \Pi+2 \Pi \partial_{z} B, \quad\left(\frac{B}{r}=\Pi\right) .
$$

Then we can obtain the conclusion through the standard $H^{m}$ estimate with Gronwall's inequality.

\section{Proof of Theorem 3: Blow-Up of Axisymmetric Hall Equations}

The proof of Theorem 3 is split into two propositions: localin-time existence of a regular solution to (14) and the finite time blow-up of the local-in-time solution.

Proposition 10. The equation

$$
\begin{aligned}
\partial_{t} \Pi & =-2 \Pi \partial_{z} \Pi \\
\Pi(x, 0) & =\Pi_{0}(x) \in H^{m}, \quad m>\frac{5}{2}
\end{aligned}
$$

has a unique local-in-time solution.
Proof. First, we find the global solution to the following regularized equation of (14) without assuming the axisymmetry,

$$
\begin{gathered}
\partial_{t} \Pi_{\epsilon}=-2 \mathscr{F}_{\epsilon}\left(\mathscr{F}_{\epsilon} \Pi_{\epsilon} \cdot \partial_{z} \mathscr{F}_{\epsilon} \Pi_{\epsilon}\right) \\
\Pi(x, 0)=\Pi_{0}(x), \quad \Pi_{0} \in H^{m}, m>\frac{5}{2}
\end{gathered}
$$

Before proceeding further, we note that the divergence theorem can be applicable due to the mollifier. Let

$$
F\left(\Pi_{\epsilon}\right)=-2 \mathscr{F}_{\epsilon}\left(\mathscr{F}_{\epsilon} \Pi_{\epsilon} \cdot \partial_{z} \mathscr{F}_{\epsilon} \Pi_{\epsilon}\right) .
$$

Hence, the image of the function $F$ defined on $H^{m}$ is included in $H^{m}$ for $m>3 / 2$.

To use the Picard theorem on $H^{m}$ space $(m>5 / 2)$, we first obtain that $F$ is Lipschitz continuous on $H^{m}$, i.e.,

$$
\begin{aligned}
\left\|F\left(\Pi_{\epsilon}^{1}\right)-F\left(\Pi_{\epsilon}^{2}\right)\right\|_{m} \\
\leq 2\left\|\mathscr{J}_{\epsilon}\left\{\mathscr{J}_{\epsilon} \Pi_{\epsilon}^{1}\left(\partial_{z} \mathscr{J}_{\epsilon} \Pi_{\epsilon}^{1}-\partial_{z} \mathscr{J}_{\epsilon} \Pi_{\epsilon}^{2}\right)\right\}\right\|_{m} \\
\quad+2\left\|\mathscr{J}_{\epsilon}\left\{\left(\mathscr{J}_{\epsilon} \Pi_{\epsilon}^{1}-\mathscr{J}_{\epsilon} \Pi_{\epsilon}^{2}\right) \partial_{z} \mathscr{J}_{\epsilon} \Pi_{\epsilon}^{2}\right\}\right\|_{m} \\
\leq 2\left\|\mathscr{J}_{\epsilon} \Pi_{\epsilon}^{1}\right\|_{m}\left\|\partial_{z} \mathscr{J}_{\epsilon}\left(\Pi_{\epsilon}^{1}-\Pi_{\epsilon}^{2}\right)\right\|_{m} \\
\quad+2\left\|\mathscr{J}_{\epsilon}\left(\Pi_{\epsilon}^{1}-\Pi_{\epsilon}^{2}\right)\right\|_{m}\left\|\partial_{z} \mathscr{J}_{\epsilon} \Pi_{\epsilon}^{2}\right\|_{m} \\
\leq \frac{C}{\epsilon^{m+1}}\left(\left\|\Pi_{\epsilon}^{1}\right\|_{L^{2}}+\left\|\Pi_{\epsilon}^{2}\right\|_{L^{2}}\right)\left\|\Pi_{\epsilon}^{1}-\Pi_{\epsilon}^{2}\right\|_{m} .
\end{aligned}
$$

$F$ is a Lipschitz continous function on a bounded open set $O$ in $H^{m}$. Now we can apply Picard theorem. For each $\epsilon>$ 0 , there exist a unique solution $\Pi_{\epsilon}$ and a finite time $T_{\epsilon}$, such that $\Pi_{\epsilon} \in C^{1}\left(0, T_{\epsilon}: H^{m}\right)$. Following the standard process of constructing local-in-time solution, we obtain an implicit form of the solution

$$
\Pi_{\epsilon}(t)=\Pi_{0}+\int_{0}^{t} F\left(\Pi_{\epsilon}(s)\right) d s, \quad 0 \leq t<T_{\epsilon} .
$$

Since $F(0)=0$, we have

$$
\begin{aligned}
&\left\|\Pi_{\epsilon}\right\|_{m}(t) \leq\left\|\Pi_{0}\right\|_{m}+\int_{0}^{t}\left\|F\left(\Pi_{\epsilon}(s)\right)\right\|_{m} d s \\
& \leq\left\|\Pi_{0}\right\|_{m}+\int_{0}^{t} C_{m, \epsilon}\left\|\Pi_{\epsilon}\right\|_{L^{2}}(s)\left\|\Pi_{\epsilon}\right\|_{m}(s) d s, \\
& \quad 0 \leq t<T_{\epsilon} .
\end{aligned}
$$

Since the above regularized equation satisfies an energy estimate, we deduce that

$$
\frac{d}{d t} \int_{\mathbb{R}^{3}} \Pi_{\epsilon}^{2} d x=-\frac{2}{3} \int_{\mathbb{R}^{3}} \partial_{z}\left(\mathscr{F}_{\epsilon} \Pi_{\epsilon}\right)^{3} d x=0,
$$

and hence

$$
\left\|\Pi_{\epsilon}\right\|_{L^{2}}(t)=\left\|\Pi_{0}\right\|_{L^{2}} .
$$

For the higher order norm, Gronwall's inequality implies

$$
\left\|\Pi_{\epsilon}\right\|_{m}(t) \leq\left\|\Pi_{0}\right\|_{m} e^{C_{m, \epsilon}\left\|\Pi_{0}\right\|_{L^{2}\left(\mathbb{R}^{3}\right)} t}, \quad 0 \leq t<T_{\epsilon} .
$$


The above inequality justifies that each solution $\Pi_{\epsilon}$ is a global solution to regularized equation, and

$$
\Pi_{\epsilon} \in C^{1}\left(0, \infty: H^{m}\right), \quad \text { for all } \Pi_{0} \in H^{m}
$$

Second, we show that, for some finite time $T$, the sequence $\left\{\Pi_{\epsilon}\right\}_{\epsilon>0}$ is a Cauchy sequence in $C\left(0, T ; L^{2}\right)$. We note that, for $T<1 / C_{m}\left\|\Pi_{0}\right\|_{m}$,

$$
\begin{gathered}
\sup _{0 \leq t \leq T}\left\|\Pi_{\epsilon}\right\|_{m}(t) \leq \frac{1}{1-C_{m} T\left\|\Pi_{0}\right\|_{m}}, \\
\sup _{0 \leq t \leq T}\left\|\partial_{t} \Pi_{\epsilon}\right\|_{m-1}(t) \leq C\left(\frac{1}{1-C_{m} T\left\|\Pi_{0}\right\|_{m}}\right)^{2} .
\end{gathered}
$$

By the standard energy estimates, we have, for $0 \leq s \leq m$,

$$
\begin{aligned}
\frac{1}{2} & \frac{d}{d t}\left\|\nabla^{s} \Pi_{\epsilon}\right\|_{L^{2}}^{2} \\
& =-\int_{\mathbb{R}^{3}} \nabla^{s}\left\{\partial_{z}\left(\mathscr{J}_{\epsilon} \Pi_{\epsilon}\right)^{2}\right\} \nabla^{s}\left(\mathscr{J}_{\epsilon} \Pi_{\epsilon}\right) d x \\
& =-\int_{\mathbb{R}^{3}} \partial_{z}\left\{\nabla^{j}\left(\mathscr{J}_{\epsilon} \Pi_{\epsilon}\right) \nabla^{s-j}\left(\mathscr{J}_{\epsilon} \Pi_{\epsilon}\right)\right\} \nabla^{s}\left(\mathscr{J}_{\epsilon} \Pi_{\epsilon}\right) d x .
\end{aligned}
$$

If $j \neq 0$ or $j \neq s$, then we obtain easily that

$$
\begin{aligned}
& \frac{1}{2} \frac{d}{d t}\left\|\nabla^{s} \Pi_{\epsilon}\right\|_{L^{2}}^{2} \\
& \leq \int_{\mathbb{R}^{3}}\left|\nabla^{j+1}\left(\mathscr{J}_{\epsilon} \Pi_{\epsilon}\right)\right|\left|\nabla^{s-j}\left(\mathscr{J}_{\epsilon} \Pi_{\epsilon}\right)\right|\left|\nabla^{s}\left(\mathscr{J}_{\epsilon} \Pi_{\epsilon}\right)\right| d x \\
& \leq C\left\|\nabla \Pi_{\epsilon}\right\|_{L^{\infty}}\left\|\Pi_{\epsilon}\right\|_{m}^{2} \leq C\left\|\Pi_{\epsilon}\right\|_{m}^{3} . \\
& \begin{aligned}
\text { If } j & =0 \text { or } j=s, \text { then we obtain } \\
\frac{1}{2} & \frac{d}{d t}\left\|\nabla^{s} \Pi_{\epsilon}\right\|_{L^{2}}^{2} \\
& =\int_{\mathbb{R}^{3}}\left(\mathscr{J}_{\epsilon} \Pi_{\epsilon}\right) \cdot \partial_{z} \nabla^{s}\left(\mathscr{J}_{\epsilon} \Pi_{\epsilon}\right) \cdot \nabla^{s}\left(\mathscr{J}_{\epsilon} \Pi_{\epsilon}\right) d x \\
& =\int_{\mathbb{R}^{3}}\left(\mathscr{J}_{\epsilon} \Pi_{\epsilon}\right) \partial_{z}\left(\nabla^{s}\left(\mathscr{J}_{\epsilon} \Pi_{\epsilon}\right)\right)^{2} d x \\
& =-\int_{\mathbb{R}^{3}} \partial_{z}\left(\mathscr{J}_{\epsilon} \Pi_{\epsilon}\right)\left|\nabla^{s}\left(\mathscr{J}_{\epsilon} \Pi_{\epsilon}\right)\right|^{2} d x \\
& \leq\left\|\partial_{z} \Pi_{\epsilon}\right\|_{L^{\infty}\left(\mathbb{R}^{3}\right)}\left\|\Pi_{\epsilon}\right\|_{m}^{2} \leq C\left\|\Pi_{\epsilon}\right\|_{m}^{3} .
\end{aligned}
\end{aligned}
$$

Combining the above inequalities (93) and (94), we have

$$
\frac{d}{d t}\left\|\Pi_{\epsilon}\right\|_{m} \leq C_{m}\left\|\Pi_{\epsilon}\right\|_{m}^{2}
$$

The above inequality gives us

$$
\left\|\Pi_{\epsilon}\right\|_{m}(t) \leq \frac{\left\|\Pi_{0}\right\|_{m}}{1-t C_{m}\left\|\Pi_{0}\right\|_{m}}
$$

$$
\text { for } 0 \leq t \leq T<\frac{1}{C_{m}\left\|\Pi_{0}\right\|_{m}} \text {, }
$$

$$
\begin{aligned}
& \sup _{0 \leq t \leq T}\left\|\Pi_{\epsilon}\right\|_{m}(t) \leq \frac{1}{1-C_{m} T\left\|\Pi_{0}\right\|_{m}} \\
& \qquad \text { for } T<\frac{1}{C_{m}\left\|\Pi_{0}\right\|_{m}} .
\end{aligned}
$$

By applying $H^{m-1}$ norm at the regularized equation, we deduce that

$$
\begin{aligned}
\left\|\partial_{t} \Pi_{\epsilon}\right\|_{m-1} & =\left\|F\left(\Pi_{\epsilon}\right)\right\|_{m-1} \leq C_{m}^{\prime}\left\|\Pi_{\epsilon}\right\|_{m-1}\left\|\partial_{z} \Pi_{\epsilon}\right\|_{m-1} \\
& \leq C_{m}^{\prime}\left\|\Pi_{\epsilon}\right\|_{m}^{2}(t) \\
& \leq C_{m}^{\prime}\left(\frac{1}{1-C_{m} T\left\|\Pi_{0}\right\|_{m}}\right)^{2} \\
& \text { for } T<\frac{1}{C_{m}\left\|\Pi_{0}\right\|_{m}} .
\end{aligned}
$$

Now we are ready to show that $\left\{\Pi_{\epsilon}\right\} \subset C\left(0, T ; L^{2}\right)$ is a Cauchy sequence (as a sequence for $\epsilon_{n} \longrightarrow 0$ ), where $T$ is chosen as above.

$$
\begin{aligned}
& \frac{1}{2} \frac{d}{d t}\left\|\Pi_{\epsilon}-\Pi_{\epsilon^{\prime}}\right\|_{L^{2}\left(\mathbb{R}^{3}\right)}^{2} \\
& =-\int_{\mathbb{R}^{3}}\left\{\mathscr{J}_{\epsilon} \partial_{z}\left(\mathscr{J}_{\epsilon} \Pi_{\epsilon}\right)^{2}-\mathscr{J}_{\epsilon^{\prime}} \partial_{z}\left(\mathscr{J}_{\epsilon^{\prime}} \Pi_{\epsilon^{\prime}}\right)^{2}\right\} \\
& \cdot\left(\Pi_{\epsilon}-\Pi_{\epsilon^{\prime}}\right) d x=-\int \mathscr{J}_{\epsilon}\left(\Pi_{\epsilon}-\Pi_{\epsilon^{\prime}}\right) \\
& \cdot \partial_{z}\left(\mathscr{J}_{\epsilon} \Pi_{\epsilon}\right)^{2}+\int \mathscr{J}_{\epsilon^{\prime}}\left(\Pi_{\epsilon}-\Pi_{\epsilon^{\prime}}\right) \partial_{z}\left(\mathscr{J}_{\epsilon^{\prime}} \Pi_{\epsilon^{\prime}}\right)^{2} \\
& \quad=-2 \int\left(\mathscr{J}_{\epsilon}-\mathscr{J}_{\epsilon^{\prime}}\right)\left(\Pi_{\epsilon}-\Pi_{\epsilon^{\prime}}\right) \mathscr{J}_{\epsilon} \Pi_{\epsilon} \partial_{z} \mathscr{J}_{\epsilon} \Pi_{\epsilon} \\
& -2 \int \mathscr{J}_{\epsilon^{\prime}}\left(\Pi_{\epsilon}-\Pi_{\epsilon^{\prime}}\right)\left(\mathscr{J}_{\epsilon} \Pi_{\epsilon}-\mathscr{J}_{\epsilon^{\prime}} \Pi_{\epsilon}\right) \partial_{z}\left(\mathscr{J}_{\epsilon} \Pi_{\epsilon}\right) \\
& \quad-2 \int\left\{\mathscr{J}_{\epsilon^{\prime}}\left(\Pi_{\epsilon}-\Pi_{\epsilon^{\prime}}\right)\right\}^{2} \partial_{z}\left(\mathscr{J}_{\epsilon} \Pi_{\epsilon}\right) \\
& \quad-2 \int \mathscr{J}_{\epsilon^{\prime}}\left(\Pi_{\epsilon}-\Pi_{\epsilon^{\prime}}\right) \mathscr{J}_{\epsilon^{\prime}} \Pi_{\epsilon^{\prime}} \partial_{z}\left(\mathscr{J}_{\epsilon} \Pi_{\epsilon}-\mathscr{J}_{\epsilon^{\prime}} \Pi_{\epsilon}\right) \\
& \quad-\int \mathscr{J}_{\epsilon^{\prime}} \Pi_{\epsilon^{\prime}} \partial_{z}\left\{\mathscr{F}_{\epsilon^{\prime}}\left(\Pi_{\epsilon}-\Pi_{\epsilon^{\prime}}\right)\right\}^{2}
\end{aligned}
$$


By the properties of regularizer $\mathscr{J}_{\epsilon}$, for $m>5 / 2$, we have

$$
\begin{aligned}
& \frac{1}{2} \frac{d}{d t}\left\|\Pi_{\epsilon}-\Pi_{\epsilon^{\prime}}\right\|_{L^{2}\left(\mathbb{R}^{3}\right)}^{2} \leq C\left\|\Pi_{\epsilon}-\Pi_{\epsilon^{\prime}}\right\|_{L^{2}}\left(\epsilon+\epsilon^{\prime}\right) \\
& \cdot\left(\left\|\partial_{z} \Pi_{\epsilon}\right\|_{L^{\infty}}\left\|\Pi_{\epsilon}\right\|_{1}+\left\|\partial_{z} \Pi_{\epsilon}\right\|_{1}\left\|\Pi_{\epsilon}\right\|_{L^{\infty}}\right)+C(\epsilon \\
& \left.+\epsilon^{\prime}\right)\left\|\Pi_{\epsilon}\right\|_{1}\left\|\Pi_{\epsilon}-\Pi_{\epsilon^{\prime}}\right\|_{L^{2}}\left\|\partial_{z} \Pi_{\epsilon}\right\|_{L^{\infty}}+C \| \Pi_{\epsilon} \\
& \quad-\Pi_{\epsilon^{\prime}}\left\|_{L^{2}}^{2}\right\| \partial_{z} \Pi_{\epsilon}\left\|_{L^{\infty}}+C\left(\epsilon+\epsilon^{\prime}\right)\right\| \Pi_{\epsilon}\left\|_{2}\right\| \Pi_{\epsilon}-\Pi_{\epsilon^{\prime}} \|_{L^{2}} \\
& \cdot\left\|\Pi_{\epsilon^{\prime}}\right\|_{L^{\infty}}+C\left\|\Pi_{\epsilon}-\Pi_{\epsilon^{\prime}}\right\|_{L^{2}}^{2}\left\|\partial_{z} \Pi_{\epsilon^{\prime}}\right\|_{L^{\infty}} \leq C(\epsilon \\
& \left.\quad+\epsilon^{\prime}\right)\left\|\Pi_{\epsilon}\right\|_{m}\left\|\Pi_{\epsilon^{\prime}}\right\|_{m}\left\|\Pi_{\epsilon}-\Pi_{\epsilon^{\prime}}\right\|_{L^{2}}+C\left(\left\|\Pi_{\epsilon}\right\|_{m}\right. \\
& \left.\quad+\left\|\Pi_{\epsilon^{\prime}}\right\|_{m}\right)\left\|\Pi_{\epsilon}-\Pi_{\epsilon^{\prime}}\right\|_{L^{2}}^{2} \leq C\left\|\Pi_{\epsilon}-\Pi_{\epsilon^{\prime}}\right\|_{L^{2}} \\
& \quad \cdot\left\{\left(\epsilon+\epsilon^{\prime}\right)\left\|\Pi_{\epsilon}\right\|_{m}\left\|\Pi_{\epsilon^{\prime}}\right\|_{m}\right. \\
& \left.\quad+\left\|\Pi_{\epsilon}-\Pi_{\epsilon^{\prime}}\right\|_{L^{2}}\left(\left\|\Pi_{\epsilon}\right\|_{m}+\left\|\Pi_{\epsilon^{\prime}}\right\|_{m}\right)\right\}
\end{aligned}
$$

In summary, we have

$$
\begin{aligned}
\frac{d}{d t}\left\|\Pi_{\epsilon}-\Pi_{\epsilon^{\prime}}\right\|_{L^{2}\left(\mathbb{R}^{3}\right)} & \\
\leq & C\left(\epsilon+\epsilon^{\prime}\right)\left\|\Pi_{\epsilon}\right\|_{m}\left\|\Pi_{\epsilon^{\prime}}\right\|_{m} \\
& +C\left\|\Pi_{\epsilon}-\Pi_{\epsilon^{\prime}}\right\|_{L^{2}}\left(\left\|\Pi_{\epsilon}\right\|_{m}+\left\|\Pi_{\epsilon^{\prime}}\right\|_{m}\right)
\end{aligned}
$$

By Gronwall's inequality, we can conclude that $\left\{\Pi_{\epsilon}\right\}$ is a Cauchy sequence in the $C\left(0, T ; L^{2}\right)$ space. And by $H^{m}$ boundness, if we apply the interpolation inequality, then we can see that $\left\{\Pi_{\epsilon}\right\}$ is a cauchy sequence in $C\left(0, T ; H^{s}\right), \forall s<m$. So we have the limit function $\Pi \in C\left(0, T ; H^{s}\right)$. And $\left\{\partial_{t} \Pi_{\epsilon}\right\}$ is also a cauchy sequence in the $C\left(0, T ; L^{2}\right)$ space by the following estimates:

$$
\begin{aligned}
\left\|\partial_{t} \Pi_{\epsilon}-\partial_{t} \Pi_{\epsilon^{\prime}}\right\|_{L^{2}} & \\
\leq & 2\left\|\mathscr{J}_{\epsilon}\left(\mathscr{J}_{\epsilon} \Pi_{\epsilon} \partial_{z} \mathscr{J}_{\epsilon} \Pi_{\epsilon}\right)-\mathscr{J}_{\epsilon^{\prime}}\left(\mathscr{J}_{\epsilon^{\prime}} \Pi_{\epsilon^{\prime}} \partial_{z} \mathscr{J}_{\epsilon^{\prime}} \Pi_{\epsilon^{\prime}}\right)\right\|_{L^{2}} \\
\leq & 2\left\|\left(\mathscr{J}_{\epsilon}-\mathscr{J}_{\epsilon^{\prime}}\right)\left(\mathscr{J}_{\epsilon} \Pi_{\epsilon} \partial_{z} \mathscr{J}_{\epsilon} \Pi_{\epsilon}\right)\right\|_{L^{2}} \\
& +2\left\|\left(\mathscr{J}_{\epsilon}-\mathscr{J}_{\epsilon^{\prime}}\right) \Pi_{\epsilon} \partial_{z} \mathscr{J}_{\epsilon} \Pi_{\epsilon}\right\|_{L^{2}} \\
& +2\left\|\mathscr{J}_{\epsilon^{\prime}}\left(\Pi_{\epsilon}-\Pi_{\epsilon^{\prime}}\right) \partial_{z} \mathscr{J}_{\epsilon} \Pi_{\epsilon}\right\|_{L^{2}} \\
& +2\left\|\mathscr{J}_{\epsilon^{\prime}} \Pi_{\epsilon^{\prime}}\left(\mathscr{J}_{\epsilon}-\mathscr{J}_{\epsilon^{\prime}}\right) \partial_{z} \Pi_{\epsilon}\right\|_{L^{2}} \\
& +2\left\|\mathscr{F}_{\epsilon^{\prime}} \Pi_{\epsilon^{\prime}} \mathscr{J}_{\epsilon^{\prime}} \partial_{z}\left(\Pi_{\epsilon}-\Pi_{\epsilon^{\prime}}\right)\right\|_{L^{2}} \\
\leq & 6\left(\epsilon+\epsilon^{\prime}\right)\left(\left\|\Pi_{\epsilon}\right\|_{s}^{2}+\left\|\Pi_{\epsilon^{\prime}}\right\|_{s}^{2}\right) \\
& +4\left(\left\|\Pi_{\epsilon}\right\|_{s}+\left\|\Pi_{\epsilon^{\prime}}\right\|_{s}\right)\left\|\Pi_{\epsilon}-\Pi_{\epsilon^{\prime}}\right\|_{1}
\end{aligned}
$$

For $5 / 2<s<m$, we have the limit function $\partial_{t} \Pi \epsilon$ $C\left(0, T ; H^{s-1}\right)$.

Finally, we can show that $\Pi \in C\left(0, T ; H^{m}\right) \cap \operatorname{Lip}(0$, $\left.T ; H^{m-1}\right)$. By the Banach Alaoglu theorem, we have $\Pi \in$ $L^{\infty}\left(0, T_{0} ; H^{m}\right)$ and $\partial_{t} \Pi \in L^{\infty}\left(0, T_{0} ; H^{m-1}\right)$, because we know that, for any $s<m, \Pi \in C\left(0, T ; H^{s}\right)$. It implies $\Pi \epsilon$ $C_{w}\left(0, T ; H^{m}\right)$ by the following estimate:

$$
\begin{aligned}
{\left[\varphi, \Pi_{\epsilon}-\Pi\right] \leq } & \|\varphi-\widetilde{\varphi}\|_{H^{-m}}\left\|\Pi_{\epsilon}-\Pi\right\|_{H^{m}} \\
& +\left[\widetilde{\varphi}, \Pi_{\epsilon}-\Pi\right] \leq \delta+\left[\widetilde{\varphi}, \Pi_{\epsilon}-\Pi\right] \\
\leq & 2 \delta
\end{aligned}
$$

for any given $\varphi \in H^{-m}$, for some $\tilde{\varphi} \in H^{-s}, s<m$. Now we show that

$$
\liminf _{t \rightarrow 0+}\|\Pi(t)\|_{m}=\limsup _{t \longrightarrow 0+}\|\Pi(t)\|_{m}=\left\|\Pi_{0}\right\|_{m} .
$$

By the weak continuity, for any $\delta>0$, there exists $r>0$ such that if $0<t<r$, then $-\delta<\left[\Pi(t)-\Pi_{0}, \varphi\right]<\delta$, for all $\varphi \in H^{-m}$. Choose $\varphi=\nabla^{2 s} \Pi_{0}$ with $s \leq m$. Then it gives us

$$
\begin{aligned}
\left\|\nabla^{s} \Pi_{0}\right\|_{L^{2}}^{2}-\delta & \leq\left\|\nabla^{s} \Pi(t)\right\|_{L^{2}}\left\|\nabla^{s} \Pi_{0}\right\|_{L^{2}} \\
& \leq \frac{\left\|\nabla^{s} \Pi(t)\right\|_{L^{2}}^{2}}{2}+\frac{\left\|\nabla^{s} \Pi_{0}\right\|_{L^{2}}^{2}}{2} .
\end{aligned}
$$

Also we have

$$
\left\|\Pi_{0}\right\|_{m}^{2} \leq\|\Pi(t)\|_{m}^{2}+2^{m+1} \delta,
$$

which implies that

$$
\liminf _{t \rightarrow 0+}\|\Pi(t)\|_{m} \geq\left\|\Pi_{0}\right\|_{m}
$$

By the $H^{m}$ boundness with weak convergence, it is deduced that

$$
\|\Pi(t)\|_{m} \leq \frac{\left\|\Pi_{0}\right\|_{m}}{1-t C_{m}\left\|\Pi_{0}\right\|_{m}}, \quad 0 \leq t \leq T
$$

which implies

$$
\underset{t \rightarrow 0+}{\limsup }\|\Pi(t)\|_{m} \leq\left\|\Pi_{0}\right\|_{m} .
$$

Thus we have the time continuity of $\Pi$ at 0 . For any $t_{0} \leq T$ and initial value $\Pi\left(t_{0}\right)$, we can obtain a right continuity at $t_{0}$ by the time translation invariant property. By the fact that $\Pi\left(-x, t_{0}-\right.$ $t$ ) is also a solution to the euqation for $0 \leq t \leq t_{0}$, we have a left continuity at $t_{0}$. Of course by the above process, the left continuity at $T$ also can be obtained. We have proved that $\Pi \epsilon$ $C\left(0, T ; H^{m}\right)$.

Proposition 11. Let $\Pi$ be an axisymmetric global classical solution to

$$
\partial_{t} \Pi+2 \Pi \partial_{z} \Pi=0
$$

Then $\Pi \equiv 0$.

Proof. Define $\phi_{r_{0}}(t):[0, \infty) \longrightarrow \mathbb{R}$ which satisfies the equation $\phi_{r_{0}}^{\prime}(t)=\Pi\left(r_{0}, 2 \phi_{r_{0}}(t), t\right)$. By our assumption, $\Pi \epsilon$ $C\left([0, \infty) ; H^{m}\left(\mathbb{R}^{3}\right)\right) \cap \operatorname{Lip}\left([0, \infty) ; H^{m-1}\left(\mathbb{R}^{3}\right)\right)$ for $m>5 / 2$, 
$\phi_{r_{0}}^{\prime \prime}(t)=0$ almost every time. So we can find the explicit form of it by

$$
\phi_{r_{0}}(t)=t \Pi_{0}\left(r_{0}, 2 \phi_{r_{0}}(0)\right)+\phi_{r_{0}}(0)
$$

Now we choose initial values $\phi_{r_{0}}(0)$ and $\widetilde{\phi}_{r_{0}}(0)$ such that $\phi_{r_{0}}(0) \leq \widetilde{\phi}_{r_{0}}(0)$. Then it satisfies

$$
\Pi_{0}\left(r_{0}, 2 \phi_{r_{0}}(0)\right) \leq \Pi_{0}\left(r_{0}, 2 \widetilde{\phi}_{r_{0}}(0)\right)
$$

Because if we suppose that $\Pi_{0}\left(r_{0}, 2 \phi_{r_{0}}(0)\right)>\Pi_{0}\left(r_{0}, 2 \widetilde{\phi}_{r_{0}}(0)\right)$, then by the explicit form of $\phi$, for some $t_{0}>0$, we have $\phi_{r_{0}}\left(t_{0}\right)=\widetilde{\phi}_{r_{0}}\left(t_{0}\right)$ which makes a contradiction. Hence, $\Pi_{0}\left(r_{0}, z\right)$ is a nondecreasing funcion with respect to $z$. Since this process is independent of the choice of $r_{0}$, we can find that $\Pi_{0} \equiv 0$ by the continuity and $L^{2}$ boundness.

\section{Proof of Theorem 5: Blow-Up Criterion}

In this section, we provide the proof of Theorem 5 which is the blow-up criterion for the axisymmetric Hall-MHD equations with $\nu=0$ and $\kappa=1$ :

$$
\begin{aligned}
& \frac{\partial \Omega}{\partial t}+\left(u^{r} \partial_{r}+u^{z} \partial_{z}\right) \Omega+2 \Pi \partial_{z} \Pi=0, \\
& \frac{\partial \Pi}{\partial t}+\left(u^{r} \partial_{r}+u^{z} \partial_{z}\right) \Pi-2 \Pi \partial_{z} \Pi \\
& =\left(\partial_{r}^{2}+\frac{3}{r} \partial_{r}+\partial_{z}^{2}\right) \Pi,
\end{aligned}
$$

where $\Omega=\omega^{\theta} / r$ and $\Pi=B^{\theta} / r$.

Known blow-up criterion for the partial viscous HallMHD equations (1) without symmetry ( $\nu=0$ and $\kappa=1)$ is as follows (see [13]).

Proposition 12. Assume that $\left(u_{0}, B_{0}\right) \in H^{m}\left(\mathbb{R}^{3}\right), m \geq 3$ with $\nabla \cdot u_{0}=\nabla \cdot B_{0}=0$. Let $(u, B)$ be a smooth solution to $(1)(v=0$ and $\kappa=1)$ for $0 \leq t<T$. If $(u, B)$ satisfies

$$
\int_{0}^{T}\left(\|\nabla \times u(t)\|_{L^{\infty}}+\|\nabla \times B(t)\|_{L^{\infty}}^{2}\right) d t<\infty
$$

then the solution $(u, B)$ can be extended beyond $t=T$.

With the axial symmetry, we can derive the following apriori estimates.

Proposition 13. If $(\Omega, \Pi)$ is a solution to (112)-(113) satisfying $(\Omega, \Pi) \in C\left([0, T) ; H^{m}\right)$, with $m \geq 3$ then it holds that

$$
\begin{aligned}
\|\Pi\|_{L^{\infty}\left(0, T ; L^{p}\left(\mathbb{R}^{3}\right)\right)} & \leq C\left(\Pi_{0}\right), \\
\|\nabla \Pi\|_{L^{2}\left(0, T ; L^{2}\left(\mathbb{R}^{3}\right)\right)}^{2} & \leq\left\|\Pi_{0}\right\|_{L^{2}}^{2}, \\
\|\Omega\|_{L^{\infty}\left(0, T ; L^{2}\right)} & \leq C\left(\left\|\Omega_{0}\right\|_{L^{2}},\left\|\Pi_{0}\right\|_{L^{2}}\right)<\infty .
\end{aligned}
$$

Proof. We first consider $p=2 n$ with $n \in \mathbb{N}$. Taking scalar product of (113) with $\Pi^{p-1}$, we deduce that

$$
\begin{aligned}
& \frac{1}{p} \frac{d}{d t}\|\Pi(t)\|_{L^{p}}^{p} \\
& \quad-2 \pi \int_{-\infty}^{\infty} \int_{0}^{\infty}\left(\left(\partial_{r}^{2}+\frac{3}{r} \partial_{r}+\partial_{z}^{2}\right) \Pi\right) \cdot \Pi^{p-1} r d r d z \\
& =\frac{2}{p+1} \int_{\mathbb{R}^{3}} \partial_{z}(\Pi)^{p+1} d x \\
& \quad-\int_{\mathbb{R}^{3}}\left(u^{r} \partial_{r}+u^{z} \partial_{z}\right) \Pi \cdot \Pi^{p-1} d x
\end{aligned}
$$

From the divergence free condition and the decay conditions like

$$
\lim _{r \longrightarrow \infty} \Pi(r, z, t)=\lim _{|z| \longrightarrow \infty} \Pi(r, z, t)=0,
$$

(116) can be reduced to

$$
\begin{gathered}
\frac{1}{p} \frac{d}{d t}\|\Pi(t)\|_{L^{p}}^{p}+\frac{4(p-1)}{p^{2}} \int_{\mathbb{R}^{3}}\left|\partial_{r} \Pi^{p / 2}\right|^{2} \\
+\left|\partial_{z} \Pi^{p / 2}\right|^{2} d x=0 .
\end{gathered}
$$

It implies that

$$
\|\Pi(t)\|_{L^{\infty}\left(0, T ; L^{p}\right)}^{p} \leq\left\|\Pi_{0}\right\|_{L^{p}}^{p} .
$$

For any $R>0$, we have

$$
\left(\int_{B_{R}}\left|\Pi_{0}(x)\right|^{2 n} d x\right)^{1 / 2 n} \leq C\left\|\Pi_{0}\right\|_{L^{\infty}} R^{3 / 2 n} .
$$

As $n \longrightarrow \infty$, we have $\|\Pi\|_{L^{\infty}\left(0, T ; L^{\infty}\right)} \leq C\left\|\Pi_{0}\right\|_{L^{\infty}}$. If $p \in$ $(2 n, 2(n+1))$, then we have

$$
\|\Pi\|_{L^{\infty}\left(0, T ; L^{p}\right)}^{p} \leq\left\|\Pi_{0}\right\|_{L^{2 n}}^{n(2 n+2-p)}\left\|\Pi_{0}\right\|_{L^{2(n+1)}}^{(n+1)(p-2 n)} .
$$

Taking $L^{2}$ scalar product of (112) with $\Omega$, we have

$$
\begin{aligned}
\frac{1}{2} \frac{d}{d t}\|\Omega\|_{L^{2}}^{2} & =-2 \int_{\mathbb{R}^{3}} \Pi \partial_{z} \Pi \Omega d x \\
& \leq 2\|\Pi\|_{L^{\infty}}\left\|\partial_{z} \Pi\right\|_{L^{2}}\|\Omega\|_{L^{2}}
\end{aligned}
$$

Then we have

$$
\begin{aligned}
\|\Omega\|_{L^{\infty}\left(0, T ; L^{2}\right)} \lesssim & \left\|\Omega_{0}\right\|_{L^{2}} \\
& +\int_{0}^{T}\|\Pi(t)\|_{L^{\infty}}^{2}+\left\|\partial_{z} \Pi(t)\right\|_{L^{2}}^{2} d t
\end{aligned}
$$

This completes the proof.

From the energy estimates of the velocity and magnetic fields, we have

$$
\begin{gathered}
\left\|u^{r}\right\|_{L^{\infty}\left(0, T ; L^{2}\right)}^{2}+\left\|u^{z}\right\|_{L^{\infty}\left(0, T ; L^{2}\right)}^{2}+\left\|B^{\theta}\right\|_{L^{\infty}\left(0, T ; L^{2}\right)}^{2} \\
+\int_{0}^{T}\left\|\partial_{r} B^{\theta}\right\|_{L^{2}}^{2}+\left\|\partial_{z} B^{\theta}\right\|_{L^{2}}^{2}+\left\|\frac{B^{\theta}}{r}\right\|_{L^{2}}^{2} d t \\
\leq\left\|u_{0}^{r}\right\|_{L^{2}}^{2}+\left\|u_{0}^{z}\right\|_{L^{2}}^{2}+\left\|B_{0}^{\theta}\right\|_{L^{2}}^{2} .
\end{gathered}
$$


Proof of Theorem 5. First, we assume that assumption (19) holds. If we consider the equation of the vorticity $\omega^{\theta}$, then we have

$$
\partial_{t} \omega^{\theta}+\left(u^{r} \partial_{r}+u^{z} \partial_{z}\right) \omega^{\theta}=\frac{u^{r}}{r} \omega^{\theta}-2\left(\frac{B^{\theta}}{r}\right) \partial_{z} B^{\theta} .
$$

Taking $L^{2}$ scalar product of (125) with $\omega^{\theta}$, we have

$$
\begin{aligned}
\frac{d}{d t}\left\|\omega^{\theta}\right\|_{L^{2}}^{2} \leq & \int_{\mathbb{R}^{3}} \frac{u_{+}^{r}}{r}\left(\omega^{\theta}\right)^{2} d x \\
& +2 \int_{\mathbb{R}^{3}}\left|\partial_{z} B^{\theta}\right||\Pi|\left|\omega^{\theta}\right| d x \\
\leq & \left\|\frac{u_{+}^{r}}{r}\right\|_{L^{\infty}\left(\mathbb{R}^{3} \backslash C_{R}\right)}\left\|\omega^{\theta}\right\|_{L^{2}\left(\mathbb{R}^{3} \backslash C_{R}\right)}^{2} \\
& +\left\|r u_{+}^{r}\right\|_{L^{\infty}\left(C_{R}\right)}\|\Omega\|_{L^{2}\left(C_{R}\right)}^{2} \\
& +2\left\|\partial_{z} B^{\theta}\right\|_{L^{2}}\|\Pi\|_{L^{\infty}}\left\|\omega^{\theta}\right\|_{L^{2}} .
\end{aligned}
$$

Using Gronwall's inequality, we have

$$
\begin{gathered}
\left\|\omega^{\theta}\right\|_{L^{\infty}\left(0, T ; L^{2}\right)} \leq\left(\left\|\omega_{0}^{\theta}\right\|_{L^{2}}+\int_{0}^{T}\left\|r u_{+}^{r}\right\|_{L^{\infty}\left(C_{R}\right)} d t+C\right) \\
\cdot \exp \left(\int_{0}^{T}\left\|\frac{u_{+}^{r}}{r}\right\|_{L^{\infty}\left(\mathbb{R}^{3} \backslash C_{R}\right)} d t+C\right) .
\end{gathered}
$$

Hence we have $\omega^{\theta} \in L^{\infty}\left(0, T ; L^{2}\right)$ if we assume (19).

If we consider the equations for $\widetilde{\nabla} \Pi\left(\widetilde{\nabla}=\left(\partial_{r}, \partial_{z}\right)\right)$, then we obtain

$$
\begin{gathered}
\partial_{t} \partial_{z} \Pi+\left(u^{r} \partial_{r}+u^{z} \partial_{z}\right) \partial_{z} \Pi \\
+\left(\partial_{z} u^{r} \partial_{r}+\partial_{z} u^{z} \partial_{z}\right) \Pi=\left(\Delta+\frac{2}{r} \partial_{r}\right) \partial_{z} \Pi+\partial_{z}^{2} \Pi^{2} \\
\partial_{t} \partial_{r} \Pi+\left(u^{r} \partial_{r}+u^{z} \partial_{z}\right) \partial_{r} \Pi+\left(\partial_{r} u^{r} \partial_{r}+\partial_{r} u^{z} \partial_{z}\right) \Pi \\
\quad+\frac{2}{r^{2}} \partial_{r} \Pi=\left(\Delta+\frac{2}{r} \partial_{r}\right) \partial_{r} \Pi+\partial_{r} \partial_{z} \Pi^{2}
\end{gathered}
$$

Via an interpolation inequality and Young's inequality, we have

$$
\begin{aligned}
& \left\|\omega^{\theta}\right\|_{L^{2}\left(\mathbb{R}^{3}\right)}\|\widetilde{\nabla} \Pi\|_{L^{6}\left(\mathbb{R}^{3}\right)}\|\widetilde{\nabla} \Pi\|_{L^{3}\left(\mathbb{R}^{3}\right)} \\
& \quad \leq\left\|\omega^{\theta}\right\|_{L^{2}\left(\mathbb{R}^{3}\right)}\left\|\widetilde{\nabla}^{2} \Pi\right\|_{L^{2}\left(\mathbb{R}^{3}\right)}^{3 / 2}\|\widetilde{\nabla} \Pi\|_{L^{2}\left(\mathbb{R}^{3}\right)}^{1 / 2} \\
& \quad \leq C\left\|\omega^{\theta}\right\|_{L^{2}\left(\mathbb{R}^{3}\right)}^{4}\|\widetilde{\nabla} \Pi\|_{L^{2}\left(\mathbb{R}^{3}\right)}^{2}+\frac{1}{2}\left\|\widetilde{\nabla}^{2} \Pi\right\|_{L^{2}\left(\mathbb{R}^{3}\right)}^{2} .
\end{aligned}
$$

Taking scalar product of (128) with $\widetilde{\nabla} \Pi$, we deduce that

$$
\begin{aligned}
& \frac{1}{2} \frac{d}{d t} \int_{\mathbb{R}^{3}}|\widetilde{\nabla} \Pi|^{2} d x+\int_{\mathbb{R}^{3}}\left|\widetilde{\nabla}^{2} \Pi\right|^{2} d x \\
& \quad+\int_{\mathbb{R}^{3}} \frac{2}{r^{2}}\left|\partial_{r} \Pi\right|^{2} d x \\
& \quad \leq C\left(\left\|\omega^{\theta}\right\|_{L^{2}}\|\widetilde{\nabla} \Pi\|_{L^{6}}\|\widetilde{\nabla} \Pi\|_{L^{3}}\right. \\
& \quad+\int_{\mathbb{R}^{3}} \frac{1}{r} \partial_{r}\left(|\widetilde{\nabla} \Pi|^{2}\right) d x+\int_{\mathbb{R}^{3}}\left|\partial_{z} \Pi\right|^{3} d x \\
& \quad+C\left\|\widetilde{\nabla}^{2} \Pi\right\|_{L^{2}}\|\widetilde{\nabla} \Pi\|_{L^{2}}\|\Pi\|_{L^{\infty}} \\
& \left.\quad+\int_{\mathbb{R}^{3}}\left|\partial_{r} \Pi\right|^{2}\left|\partial_{z} \Pi\right| d x\right) \leq C\left(\left\|\omega^{\theta}\right\|_{L^{2}}^{4}+\|\widetilde{\nabla} \Pi\|_{L^{2}}^{2}\right. \\
& \left.\quad+\|\Pi\|_{L^{\infty}}^{2}\right)\|\tilde{\nabla} \Pi\|_{L^{2}}^{2}+\frac{1}{2}\|\widetilde{\nabla} \Pi\|_{L^{2}}^{2} .
\end{aligned}
$$

In the above, we used the fact that $(1 / r) \partial_{r}\left(\left|\partial_{r} \Pi\right|^{2}+\left|\partial_{z} \Pi\right|^{2}\right) \epsilon$ $L^{1}\left(\mathbb{R}^{3}\right)$ when $t<T^{*}$. Gronwall's inequality again gives us

$$
\begin{gathered}
\widetilde{\nabla} \Pi \in L^{\infty}\left(0, T ; L^{2}\right), \\
\widetilde{\nabla}^{2} \Pi \in L^{2}\left(0, T ; L^{2}\right) .
\end{gathered}
$$

Multiplying both sides of (112) with $|\Omega|^{4} \Omega$ and integrating over $\mathbb{R}^{3}$, we have

$$
\begin{aligned}
\frac{1}{6} \frac{d}{d t}\|\Omega\|_{L^{6}\left(\mathbb{R}^{3}\right)}^{6} & \leq 2 \int_{\mathbb{R}^{3}}\left|\partial_{z} \Pi\right||\Pi||\Omega|^{5} d x \\
& \leq\|\widetilde{\nabla} \Pi\|_{L^{6}}\|\Pi\|_{L^{\infty}}\|\Omega\|_{6}^{5} .
\end{aligned}
$$

Then it is immediate that $\Omega \in L^{\infty}\left(0, T ; L^{6}\right)$.

Following the ideas in [18], we introduce the angular stream function $\psi^{\theta}$ such that

$$
-\left(\partial_{r}^{2}+\frac{1}{r} \partial_{r}-\frac{1}{r^{2}}+\partial_{z}^{2}\right) \psi^{\theta}=\omega^{\theta} .
$$

For all $1<p<\infty$, we have

$$
\int\left|\nabla^{2} \frac{\psi^{\theta}}{r}\right|^{p} d x \leq C \int\left|\frac{\omega^{\theta}}{r}\right|^{p} d x .
$$

By the interpolation inequality $\|f\|_{L^{\infty}} \leq C\|\nabla f\|_{L^{2}}^{1 / 2}\|\nabla f\|_{L^{6}}^{1 / 2}$, we have

$$
\begin{aligned}
& \int_{0}^{T}\left\|\frac{u^{r}}{r}\right\|_{L^{\infty}} d t \\
& \quad \leq \int_{0}^{T}\left\|\nabla \partial_{z}\left(\frac{\psi^{\theta}}{r}\right)\right\|_{L^{2}}^{1 / 2}\left\|\nabla \partial_{z}\left(\frac{\psi^{\theta}}{r}\right)\right\|_{L^{6}}^{1 / 2} d t \\
& \quad \leq \sup _{t}\|\Omega\|_{L^{2}}^{1 / 2} \int_{0}^{T}\|\Omega\|_{L^{6}}^{1 / 2} d t .
\end{aligned}
$$


Therefore, we have $u^{r} / r \in L^{1}\left(0, T ; L^{\infty}\right)$. Also we can have $u^{r} / r \in L^{p}\left(0, T ; L^{\infty}\right)$ for all $p<\infty$.

If we multiply $\left(B^{\theta}\right)^{2 n-1}$ on the both sides of $(9)_{4}$ and integrate over $\mathbb{R}^{3}$, then we have

$$
\begin{gathered}
\frac{1}{2 n} \frac{d}{d t}\left\|B^{\theta}\right\|_{L^{2 n}}^{2 n}+\frac{(2 n-1)}{n^{2}}\left\|\tilde{\nabla}\left|B^{\theta}\right|^{n}\right\|_{L^{2}}^{2}+\left\|\frac{\left(B^{\theta}\right)^{n}}{r}\right\|_{L^{2}}^{2} \\
=\int_{\mathbb{R}^{3}} \frac{u^{r}}{r}\left(B^{\theta}\right)^{2 n} d x \\
\quad+2 \pi \int_{0}^{\infty} \int_{-\infty}^{\infty} \partial_{z}\left(B^{\theta}\right)^{2}\left(B^{\theta}\right)^{2 n-1} d z d r \\
\leq\left\|\frac{u^{r}}{r}\right\|_{L^{\infty}}\left\|B^{\theta}\right\|_{L^{2 n}}^{2 n} .
\end{gathered}
$$

Hence we deduce that

$$
\left\|B^{\theta}\right\|_{L^{\infty}\left(0, T ; L^{2 n}\right)} \leq\left\|B_{0}^{\theta}\right\|_{L^{2 n}} \exp \left(\int_{0}^{T}\left\|\frac{u^{r}}{r}\right\|_{L^{\infty}} d t\right) .
$$

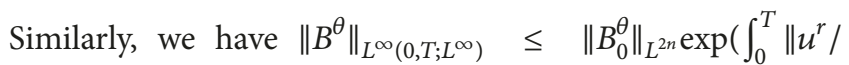
$\left.r \|_{L^{\infty}} d t\right)$.

Setting $\mathbf{B}=B^{\theta} e_{\theta}$, we have

$$
\partial_{t} \mathbf{B}+(u \cdot \nabla) \mathbf{B}-\Delta \mathbf{B}=\frac{u^{r}}{r} \mathbf{B}+2 \partial_{z} \mathbf{B} \Pi .
$$

By the maximal inequality, we have

$$
\begin{gathered}
\|\Delta \mathbf{B}\|_{L_{x, t}^{4,4}\left(\mathbb{R}^{3} \times(0 . T)\right)}^{4} \leq C\left(\left\|\mathbf{B}_{0}\right\|_{W^{2,4}}^{4}\right. \\
+\|u\|_{L_{x, t}^{6, \infty}\left(\mathbb{R}^{3} \times(0 . T)\right)}^{4}\|\nabla \mathbf{B}\|_{L_{x, t}^{12,4}\left(\mathbb{R}^{3} \times(0 . T)\right)}^{4} \\
+\left\|\frac{u^{r}}{r}\right\|_{L_{x, t}^{\infty, 4}\left(\mathbb{R}^{3} \times(0 . T)\right)}^{4}\|\mathbf{B}\|_{L_{x, t}^{4, \infty}\left(\mathbb{R}^{3} \times(0 . T)\right)}^{4} \\
\left.+\|\Pi\|_{L_{x, t}^{\infty, \infty \infty}\left(\mathbb{R}^{3} \times(0 . T)\right)}^{4}\|\nabla \mathbf{B}\|_{L_{x, t}^{4,4}\left(\mathbb{R}^{3} \times(0 . T)\right)}^{4}\right) .
\end{gathered}
$$

By the Gagliardo-Nirenberg inequality

$$
\begin{gathered}
\|\nabla \mathbf{B}\|_{L^{12}} \leq C\|\mathbf{B}\|_{L^{\infty}}^{2 / 5}\|\Delta \mathbf{B}\|_{L^{4}}^{3 / 5}, \\
\|\nabla \mathbf{B}\|_{L^{4}} \leq C\|\mathbf{B}\|_{L^{\infty}}^{4 / 5}\|\Delta \mathbf{B}\|_{L^{4}}^{1 / 5},
\end{gathered}
$$

(139) can be reduced to

$$
\begin{aligned}
& \|\Delta \mathbf{B}\|_{L_{x, t}^{4,4}\left(\mathbb{R}^{3} \times(0 . T)\right)}^{4} \leq C\left(\left\|\mathbf{B}_{\mathbf{0}}\right\|_{W^{2,4}}^{4}+\|\mathbf{B}\|_{L_{x, t}^{4, \infty}\left(\mathbb{R}^{3} \times(0 . T)\right)}^{4}\right.
\end{aligned}
$$

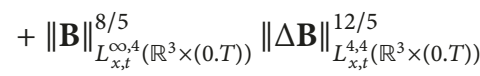

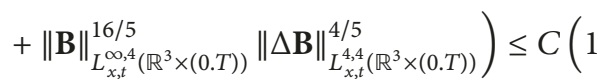

$$
\begin{aligned}
& \left.+\|\mathbf{B}\|_{L_{x, t}^{4, \infty}\left(\mathbb{R}^{3} \times(0 . T)\right)}^{4}+\|\mathbf{B}\|_{L_{x, t}^{\infty, 4}\left(\mathbb{R}^{3} \times(0 . T)\right)}^{4}\right) \\
& +\frac{1}{2}\|\Delta \mathbf{B}\|_{L_{x, t}^{4,4}\left(\mathbb{R}^{3} \times(0 . T)\right)}^{4} .
\end{aligned}
$$

Since the last term in the above can be absorbed in the left hand side, we have

$$
\begin{aligned}
& \Delta \mathbf{B} \in L^{4}\left(0, T ; L^{4}\right), \\
& \nabla \mathbf{B} \in L^{4}\left(0, T ; L^{\infty}\right) .
\end{aligned}
$$

Then, from (125), we have, for all $1<p<\infty$,

$$
\begin{aligned}
\frac{1}{p} \frac{d}{d t}\left\|\omega^{\theta}\right\|_{L^{p}}^{p} \leq & \left\|\frac{u^{r}}{r}\right\|_{L^{\infty}}\left\|\omega^{\theta}\right\|_{L^{p}}^{p} \\
& +2\|\Pi\|_{L^{\infty}}\left\|\nabla B^{\theta}\right\|_{L^{p}}\left\|\omega^{\theta}\right\|_{L^{p}}^{p-1} .
\end{aligned}
$$

By Gronwall's inequality, we have

$$
\begin{aligned}
& \left\|\omega^{\theta}\right\|_{L^{\infty}\left(0, T ; L^{p}\right)} \leq\left(\left\|\omega_{0}^{\theta}\right\|_{L^{p}}+2 \int_{0}^{T}\|\Pi\|_{L^{\infty}}\left\|\nabla B^{\theta}\right\|_{L^{p}} d t\right) \\
& \cdot \exp \left(\int_{0}^{T}\left\|\frac{u^{r}}{r}\right\|_{L^{\infty}} d t\right) .
\end{aligned}
$$

If we let $p \longrightarrow \infty$, we obtain $\omega^{\theta} \in L^{\infty}\left(0, T ; L^{\infty}\right)$. Hence, for any $T<\infty$, we obtain that $\omega^{\theta}, \nabla \mathbf{B} \in L^{2}\left(0, T ; L^{\infty}\right)$ and conclude that there does not exist a finite time blow-up if we assume (19).

Next, we assume that condition (20) holds. If we apply (129) to (128), we obtain

$$
\begin{aligned}
& \frac{1}{2} \frac{d}{d t} \int_{\mathbb{R}^{3}}|\widetilde{\nabla} \Pi|^{2} d x+\int_{\mathbb{R}^{3}}\left|\widetilde{\nabla}^{2} \Pi\right|^{2} d x \\
& \quad+\int_{\mathbb{R}^{3}} \frac{2}{r^{2}}\left|\partial_{r} \Pi\right|^{2} d x \leq C\left(\left\|\omega^{\theta}\right\|_{L^{2}\left(\mathbb{R}^{3}\right)}^{4}\|\bar{\nabla} \Pi\|_{L^{2}\left(\mathbb{R}^{3}\right)}^{2}\right. \\
& \quad+\frac{1}{2}\left\|\widetilde{\nabla}^{2} \Pi\right\|_{L^{2}\left(\mathbb{R}^{3}\right)}^{2}+\int_{\mathbb{R}^{3}} \frac{1}{r} \partial_{r}\left(\left|\partial_{r} \Pi\right|^{2}+\left|\partial_{z} \Pi\right|^{2}\right) d x \\
& \quad+\int_{\mathbb{R}^{3}}\left|\partial_{z} \Pi\right|^{3} d x+\int_{\mathbb{R}^{3}}\left|\partial_{z}^{2} \Pi\right|\left|\partial_{z} \Pi\right||\Pi| d x \\
& \left.\quad+\int_{\mathbb{R}^{3}}\left|\partial_{r} \Pi\right|^{2}\left|\partial_{z} \Pi\right| d x+\int_{\mathbb{R}^{3}}\left|\widetilde{\nabla}^{2} \Pi\right|\left|\partial_{r} \Pi\right||\Pi| d x\right)
\end{aligned}
$$

\section{Using an inequality}

$$
\left\|\omega^{\theta}\right\|_{L^{2}\left(\mathbb{R}^{3}\right)}^{4} \leq 2\left\|\omega^{\theta}\right\|_{L^{2}\left(\mathbb{R}^{3} \backslash C_{R}\right)}^{4}+2 R^{4}\|\Omega\|_{L^{2}\left(C_{R}\right)}^{4},
$$


we have

$$
\begin{aligned}
& \frac{1}{2} \frac{d}{d t} \int_{\mathbb{R}^{3}}|\widetilde{\nabla} \Pi|^{2} d x+\int_{\mathbb{R}^{3}}\left|\widetilde{\nabla}^{2} \Pi\right|^{2} d x \\
& +\int_{\mathbb{R}^{3}} \frac{2}{r^{2}}\left|\partial_{r} \Pi\right|^{2} d x \\
& \leq C\left(\left\|\omega^{\theta}\right\|_{L^{2}\left(\mathbb{R}^{3} \backslash C_{R}\right)}^{4}+R^{4}\|\Omega\|_{L^{2}\left(C_{R}\right)}^{4}\right)\|\tilde{\nabla} \Pi\|_{L^{2}\left(\mathbb{R}^{3}\right)}^{2} \\
& +\frac{1}{2}\left\|\widetilde{\nabla}^{2} \Pi\right\|_{L^{2}\left(\mathbb{R}^{3}\right)}^{2} \\
& +\int_{\mathbb{R}^{3}} \frac{1}{r} \partial_{r}\left(\left|\partial_{r} \Pi\right|^{2}+\left|\partial_{z} \Pi\right|^{2}\right) d x \\
& +\int_{\mathbb{R}^{3}}\left|\partial_{z} \Pi\right|^{3} d x+\int_{\mathbb{R}^{3}}\left|\partial_{z}^{2} \Pi\right|\left|\partial_{z} \Pi\right||\Pi| d x \\
& +\int_{\mathbb{R}^{3}}\left|\partial_{r} \Pi\right|^{2}\left|\partial_{z} \Pi\right| d x \\
& +\int_{\mathbb{R}^{3}}\left|\widetilde{\nabla}^{2} \Pi\right|\left|\partial_{r} \Pi\right||\Pi| d x .
\end{aligned}
$$

By Gronwall's inequality, we conclude that

$$
\begin{gathered}
\tilde{\nabla} \Pi \in L^{\infty}\left(0, T ; L^{2}\right), \\
\widetilde{\nabla}^{2} \Pi \in L^{2}\left(0, T ; L^{2}\right) .
\end{gathered}
$$

The estimate of $\Omega, u^{r} / r, \mathbf{B}$, and $\widetilde{\nabla} \Pi$ can be obtained similarly to the proof of the condition of (19). This completes the proof.

\section{Data Availability}

No data were used to support this study.

\section{Conflicts of Interest}

The authors declare that they have no conflicts of interest.

\section{Acknowledgments}

E. Jeong was partially supported by Chung-Ang University Excellent Student Scholarship and J. Kim and J. Lee were partially supported by NRF Grant No. 2016R1A2B3011647. The authors thank Professor Dongho Chae for helpful remarks.

\section{References}

[1] T. G. Forbes, "Magnetic Reconnection in Solar Flares," Geophysical \& Astrophysical Fluid Dynamics, vol. 62, no. 1-4, pp. 15-36, 1991.

[2] M. J. Lighthill, "Studies on magneto-hydrodynamic waves and other anisotropic wave motions," Philosophical Transactions of the Royal Society of London. Series A. Mathematical and Physical Sciences, vol. 252, pp. 397-430, 1960.

[3] P. A. Sturrock, Plasma Physics, Cambridge University Press, 1994.
[4] S. A. Balbus and C. Terquem, "Linear analysis of the hall effect in protostellar disks," The Astrophysical Journal, vol. 552, no. 1, pp. 235-247, 2001.

[5] H. Homann and R. Grauer, "Bifurcation analysis of magnetic reconnection in Hall-MHD-systems," Physica D: Nonlinear Phenomena, vol. 208, no. 1-2, pp. 59-72, 2005.

[6] P. D. Mininni, D. O. Gómez, and S. M. Mahajan, "Dynamo action in magnetohydrodynamics and Hall-magnetohydrodynamics," The Astrophysical Journal, vol. 587, no. 1 I, pp. 472-481, 2003.

[7] D. A. Shalybkov and V. A. Urpin, "The Hall effect and the decay of magnetic fields," Astron. Astrophys, vol. 32, pp. 685-690, 1997.

[8] M. Wardle, "Star formation and the hall effect," Astrophysics and Space Science, vol. 292, no. 1-4, pp. 317-323, 2004.

[9] M. Acheritogaray, P. Degond, A. Frouvelle, and J. Liu, "Kinetic formulation and global existence for the Hall-Magnetohydrodynamics system," Kinetic and Related Models, vol. 4, no. 4, pp. 901-918, 2011.

[10] D. Chae, P. Degond, and J. Liu, "Well-posedness for Hallmagnetohydrodynamics," Annales de l'Institut Henri Poincaré (C) Analyse Non Linéaire, vol. 31, no. 3, pp. 555-565, 2014.

[11] D. Chae and J. Lee, "On the blow-up criterion and small data global existence for the Hall-magnetohydrodynamics," Journal of Differential Equations, vol. 256, no. 11, pp. 3835-3858, 2014.

[12] M. Dai, "Regularity criterion for the 3D Hall-magnetohydrodynamics," Journal of Differential Equations, vol. 261, no. 1, pp. 573-591, 2016.

[13] Y.-Z. Wang and W. Zuo, "On the blow-up criterion of smooth solutions for Hall-magnetohydrodynamics system with partial viscosity," Communications on Pure and Applied Analysis, vol. 13, no. 3, pp. 1327-1336, 2014.

[14] D. Chae and M. Schonbek, "On the temporal decay for the Hall-magnetohydrodynamic equations," Journal of Differential Equations, vol. 255, no. 11, pp. 3971-3982, 2013.

[15] S. Weng, "On analyticity and temporal decay rates of solutions to the viscous resistive Hall-MHD system," Journal of Differential Equations, vol. 260, no. 8, pp. 6504-6524, 2016.

[16] O. A. Ladyzhenskaya, "Unique global solvability of the threedimensional Cauchy problem for the Navier-Stokes equations in the presence of axial symmetry," Zap. Nauchn. Sem. Leningrad. Otdel. Mati. Inst. Steklov. (LOMI), vol. 7, pp. 155-177, 1968.

[17] M. R. Ukhovskii and V. I. Iudovich, "Axially symmetric flows of ideal and viscous fluids filling the whole space," Journal of Applied Mathematics and Mechanics, vol. 32, pp. 52-61, 1968.

[18] Z. Lei, "On axially symmetric incompressible magnetohydrodynamics in three dimensions," Journal of Differential Equations, vol. 259, no. 7, pp. 3202-3215, 2015.

[19] J. Fan, S. Huang, and G. Nakamura, "Well-posedness for the axisymmetric incompressible viscous Hall-magnetohydrodynamic equations," Applied Mathematics Letters, vol. 26, no. 9, pp. 963-967, 2013.

[20] D. Chae and S. Weng, "Singularity formation for the incompressible Hall-MHD equations without resistivity," Ann. Inst. Henri Poincare-Analyse Nonlineaire, vol. 33, no. 4, pp. 10091022, 2016.

[21] D. Chae, R. Wan, and J. Wu, "Local well-posedness for the HallMHD equations with fractional magnetic diffusion," Journal of Mathematical Fluid Mechanics, vol. 17, no. 4, pp. 627-638, 2015.

[22] A. J. Majda and A. L. Bertozzi, Vorticity and Incompressible Flow, Cambridge University Press, Cambridge, UK, 2002. 


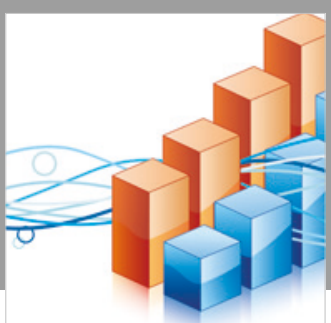

Advances in

Operations Research

\section{-n-m}
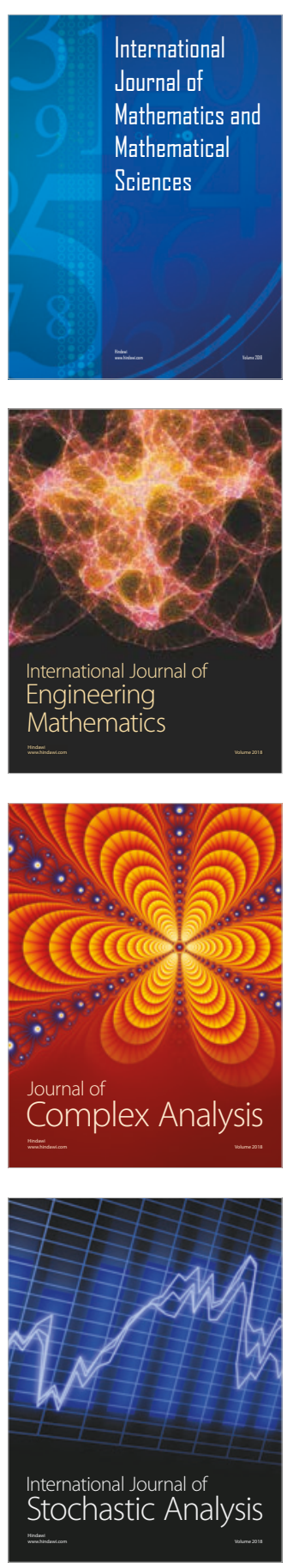
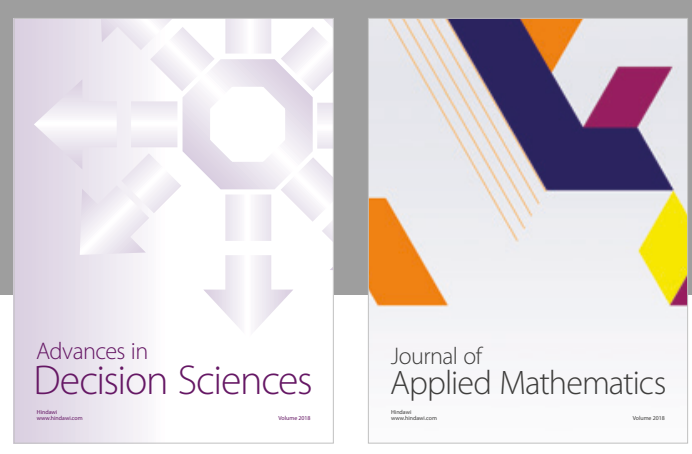

Journal of

Applied Mathematics
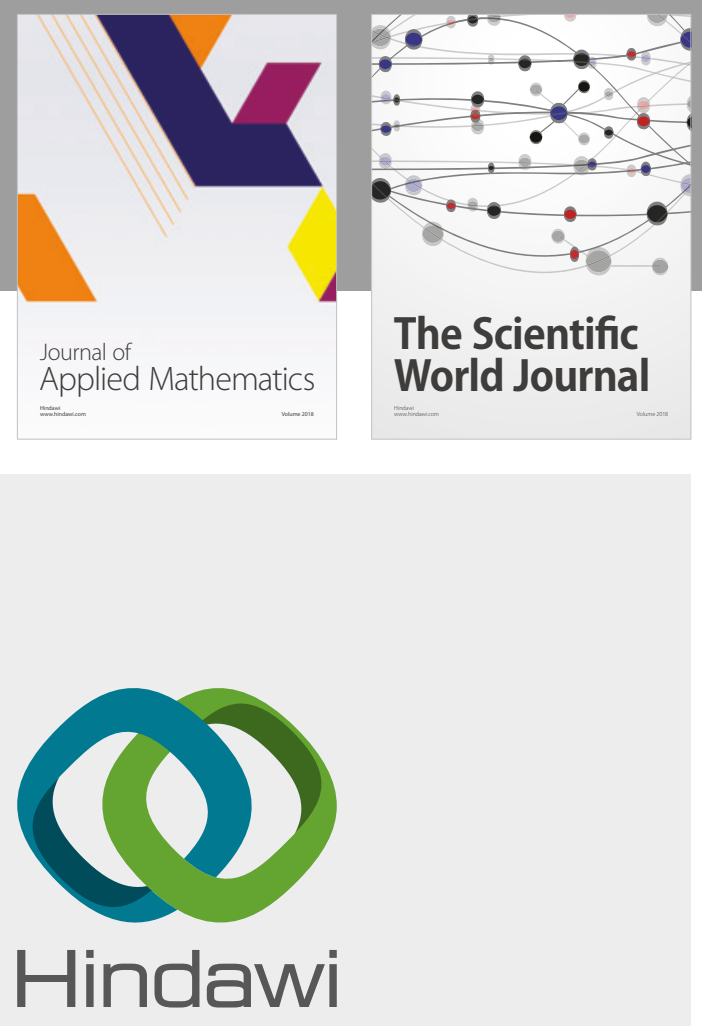

Submit your manuscripts at

www.hindawi.com

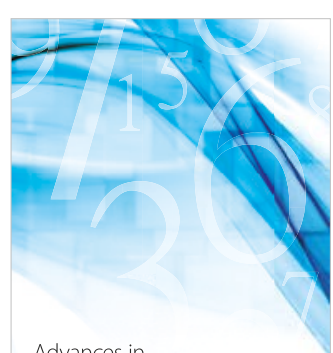

Advances in
Numerical Analysis
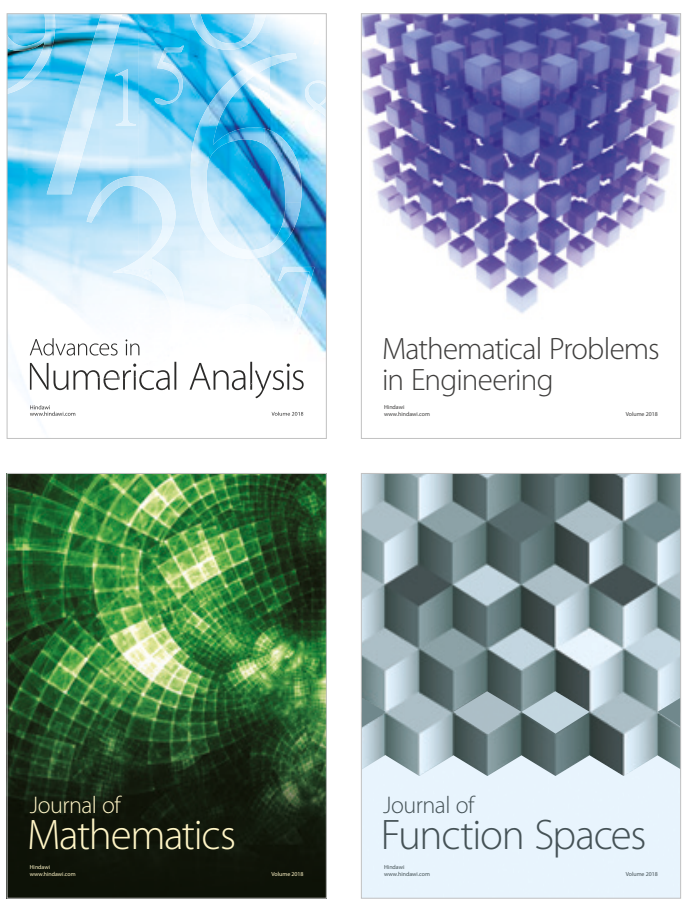

Mathematical Problems in Engineering

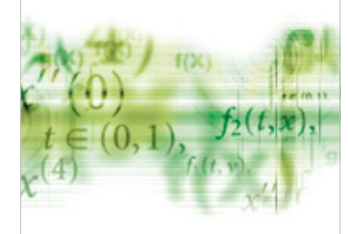

International Journal of

Differential Equations

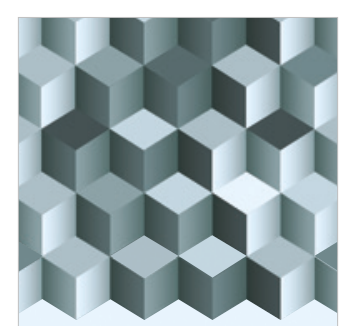

Journal of

Function Spaces

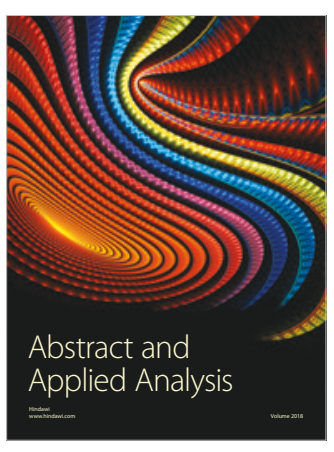

The Scientific

World Journal

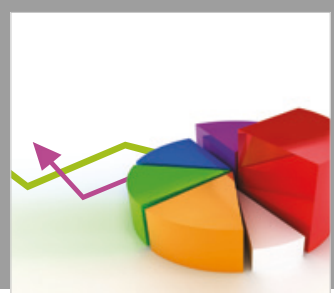

Journal of

Probability and Statistics
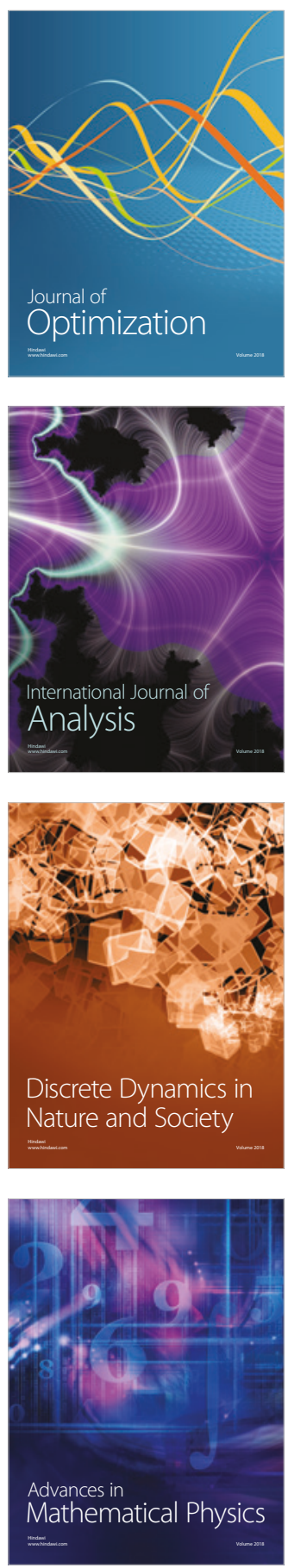